\title{
Evaluation of long-dated investments under uncertain growth trend, volatility and catastrophes
}

\author{
Christian Gollier ${ }^{1}$ \\ Toulouse School of Economics (LERNA, University of Toulouse)
}

November 2012

\begin{abstract}
Because of the uncertainty about how to model the growth process of our economy, there is still much confusion about which discount rates should be used to evaluate actions having long-lasting impacts, as in the contexts of climate change, social security reforms or large public infrastructures for example. We characterize efficient discount rates when the growth of log consumption follows a random walk with uncertain parameters. We examine different models in which the parametric uncertainty affects the trend and the volatility of growth, or the frequency of catastrophes. This uncertainty implies that the term structures of the risk free discount rate and of the aggregate risk premium are respectively decreasing and increasing. It also implies that the discount rate is increasing with maturity if the beta of the investment is larger than half of relative risk aversion. Another important consequence of parametric uncertainty is that the risk premium is not proportional to the beta of the investment. Finally, we apply our findings to the evaluation of climate change policy. We argue in particular that the beta of actions to mitigate climate change is relatively large, so that the term structure of the associated discount rates should be increasing.
\end{abstract}

Keywords: asset prices, term structure, risk premium, decreasing discount rates, parametric uncertainty, $\mathrm{CO} 2$ beta, rare events, macroeconomic catastrophes.

JEL Codes: G11, G12, E43, Q54.

\footnotetext{
${ }^{1}$ I am indebted to Marty Weitzman and Jim Hammitt for useful discussions and helpful comments on this paper during my research visit at the Harvard economics department in the Fall semester of 2012. I also thank Stéphane Gallon for useful comments on an earlier version of this paper. The research leading to these results has received funding from the Chairs "Risk Markets and Value Creation” and "Sustainable Finance and Responsible Investments" at TSE, and from the European Research Council under the European Community's Seventh Framework Programme (FP7/2007-2013) Grant Agreement no. 230589.
} 


\section{Introduction}

Do we do enough for the distant future? This question is implicit in many policy debates, from the fight against climate change to the speed of reduction of public deficits, investments in research and education, or the protection of the environment and of natural resources for example. The discount rate used to evaluate investments is the key determinant of our individual and collective efforts in favor of the future. Since Weitzman (1998), an intense debate has emerged among economists about whether one should use different discount rates for different time horizons $t$. It is however well-known that the term structure of efficient discount rates is flat if we assume that the representative agent has a constant relative risk aversion and that the growth rate of consumption follows a random walk. In this benchmark specification, if a rate of $3 \%$ is efficient to discount cash flows occurring in 12 months, it is also efficient to use that rate of $3 \%$ to discount cash flows occurring in 200 years. This yields an exponentially decreasing present value of a given benefit as a function of its maturity.

Compared to this benchmark, a decreasing term structure of discount rates would bias the economic evaluation of investments towards those with more distant benefits. Weitzman (1998, 2001) and Newell and Pizer (2003) justified such a decreasing structure by relying on the observation that the return of capital is risky. Gollier (2002, 2008, 2012) and Weitzman (2007) used standard consumption-based asset pricing theory to explore the same question. The basic idea is that the large uncertainty associated to aggregate consumption in the distant future should induce the prudent representative agent to use lower rates to discount more distant cash flows. ${ }^{2}$ Under constant relative risk aversion (CRRA), the various dynamic processes that support this result include for example mean-reversion, Markov regime-switches, and parametric uncertainty on the trend of a Brownian motion. ${ }^{3}$ Gollier (2008) demonstrates that the positive serial correlation (or persistence) of the growth of consumption that is inherent to these stochastic processes is the driving force of the result, together with prudence. For growth processes with persistent shocks, aggregate uncertainty accumulates faster with respect to longer time horizons

\footnotetext{
${ }^{2}$ Prudence is a concept defined by Kimball (1990) to characterize the willingness to save more when the future becomes more uncertain.

${ }^{3}$ Persistent movements in expected growth rates of aggregate consumption are documented for the U.S. by Bansal and Yaron (2004) for example.
} 
than in a pure random walk with the same instantaneous volatility. Prudent people want to bias their investments towards those which yield more sure benefits for these horizons. Because the term structure of socially efficient discount rates is flat under a random walk, this bias is implemented by using a decreasing term structure.

With the notable exception of Weitzman (2012), this recent literature focuses on rates $r_{f t}$ at which safe cash flows should be discounted. In reality, most investment projects yield uncertain future costs and benefits. For marginal projects, we know that idiosyncratic risks should not be priced, because they will be washed out in diversified portfolios. In public economics, this result is usually referred to as the Arrow-Lind Theorem (Arrow and Lind (1970)), but this is a wellknown feature of the consumption-based capital asset pricing model (CCAPM, Lucas (1978)). More generally, the discount rate $\rho_{t}$ to be used to evaluate risky projects depends upon their beta which measures the elasticity of net cash flows to changes in aggregate consumption. A positive beta justifies a positive risk premium $\pi_{t}(\beta)=\rho_{t}(\beta)-r_{f t}$. As is well-known in the CCAPM, in the benchmark specification with CRRA and a random walk for the growth rate of consumption, the term structure of the risk premium is flat. However, the arguments listed above in favor of a decreasing term structure of safe discount rates are also compatible with an increasing term structure of the risk premium associated to projects with a positive beta. If we assume that the stochastic process of the growth rate of consumption exhibits positive serial correlation, the annualized measure of aggregate risk will have an increasing term structure. Under risk aversion, the term structure of the risk premium will inherit this property.

With positively serially correlated growth rates, the project-specific discount rate $\rho_{t}(\beta)=r_{f t}+\pi_{t}(\beta)$ for positive betas is thus the sum of a prudence-driven decreasing function $r_{f t}$ and of a risk-aversion-driven increasing function $\pi_{t}(\beta)$ of the time horizon $t$. In standard models, this risk premium is proportional to the beta. Thus, this term structure will be decreasing if the project-specific beta is small enough, otherwise it will be increasing. This implies that the recent literature on the discount rate that has been advocating for decreasing discount rates is potentially misleading. If the beta of some green projects is positive and large, $\rho_{t}(\beta)$ may well be increasing with maturity. This paper provides a more balanced discussion about the shape of the term structure of discount rates. 
For maturities measured in decades and centuries, we believe that it is crucial to adapt the CCAPM by recognizing that the stochastic process governing the growth of aggregate consumption is affected by parametric uncertainties. We consider two alternative specifications. Under the first specification, which is examined in Section 3, we assume that log consumption follows an arithmetic Brownian motion conditional to knowing the true trend and volatility, but we assume that these parameters of the model are uncertain. Observe that the uncertainty on the trend of growth implies that the unconditional growth rates are positively correlated, thereby generating the phenomena described above. This analysis in generalized to the case of meanreversion in Section 4.

A second specification is examined in Section 5, in which we assume that the economy may face macroeconomic catastrophes at low frequency. In normal time, the growth of log consumption is Gaussian, but a large drop in aggregate consumption strikes the economy at infrequent dates. Our modeling duplicates the one proposed by Barro (2006, 2009), at the notable exception that we take seriously a critique formulated by Martin (2012). Martin convincingly demonstrates that it is extremely complex to estimate the true probability of infrequent catastrophes, and that a small modification in the choice of parameters values has a huge effect on asset prices. We take this into account by explicitly introducing ambiguity about this probability into the model. This implies the same kind of positive serial correlation in the unconditional growth rates of the economy. An important consequence of introducing uncertainty on the probability of catastrophes is that, contrary to Barro (2006, 2009), the term structures of discount rates are generally not flat. We show in Section 5 that the risk free rate and of the risk premium are respectively decreasing and increasing.

The method used in this paper was suggested by Martin (2012). We followed here this author's suggestion to use the properties of Cumulant-Generating Functions (CGF) to characterize asset prices when the growth of log consumption is not Gaussian. This paper provides various illustrations of the power of this method, whose basic elements are presented in Section 2. It can also be inferred from Martin (2012) that abandoning the Gaussian assumption for growth introduces a new source of complexity in asset pricing theory. Namely, without the Gaussian assumption, the risk premium is generally not proportional to the beta of the asset. It implies that 
knowing the aggregate risk premium and the asset's beta is not enough anymore to determine the risk premium associated to the asset. We show in this paper that the nonlinearity is quite impressive in realistic calibrations of our specifications.

Our paper provides important new insights about how public policies should be evaluated around the world. It is worrying to observe that it is common practice in public administrations around the world to use a single discount rate to evaluate public investments independent of their riskiness and time horizons. In the U.S. for example, the Office of Management and Budget (OMB) recommend to use a flat discount rate of 7\% since 1992. It was argued that the "7\% is an estimate of the average before-tax rate of return to private capital in the U.S. economy" (OMB (2003)). In 2003, the OMB also recommended the use of a discount rate of $3 \%$, in addition to the $7 \%$ mentioned above as a sensitivity. The $3 \%$ corresponds to the average real rate of return of the relatively safe 10-year Treasury notes between 1973 and 2003. Interestingly enough, the recommended use of $3 \%$ and $7 \%$ is not differentiated by the nature of the underlying risk, and is independent of the time horizon of the project. In another field, guidelines established by the Government Accounting Standards Board (GASB) recommend that state and local governments discount their pension liabilities at expected returns on their plan assets, which is usually estimated around $8 \%$, independent of their maturities. ${ }^{4}$ The absence of risk-and-maturity-based price signals has potentially catastrophic consequences for the allocation of capital in the economy. ${ }^{5}$ This paper provides clear recommendations about the changes in evaluation tools that should be implemented.

Another aim in this paper is to make recommendations about which discount rates should be used to evaluate environmental policies, in particular those associated to climate change. This raises the question of the beta of climate change, which we believe to be crucial for the determination of the so-called "social cost of carbon” (SCC). Sandsmark and Vennemo (2007) claim that the beta of mitigation investments is negative, so that the term structure of discount rates should be low and decreasing, thereby yielding a large SCC. They consider a simplified version of the standard

\footnotetext{
${ }^{4}$ The European Union is currently debating about the new solvency regulation of insurance companies (Solvency 2). In the most recent consultation paper (European Insurance and Occupational Pensions Authority (2012)), it is proposed to discount safe liabilities using the yield curve up to 20-year maturities, and a real discount rate tending to 2\% ("Ultimate Forward Rate") for longer maturities.

${ }^{5}$ In 2005, France has adopted a decreasing real discount rate from $4 \%$ to $2 \%$ for safe projects. This rule has been complemented in 2011 by an aggregate risk premium of 3\% (Gollier (2011)).
} 
integrated assessment model by Nordhaus’ DICE model (Nordhaus and Boyer (2000)). They assumed that the only source of aggregate fluctuations originates from climate change, with an uncertain climate sensitivity affecting socioeconomic damages to the economy. ${ }^{6}$ Under this assumption, a large climate sensitivity yields at the same time a low consumption (due to the climate damages) and a large social benefit from early mitigation. This explains the negative beta of their model. But suppose alternatively that the climate sensitivity is known, but the growth rate of aggregate consumption is unknown. Because emissions are increasing in consumption, a larger growth rate of consumption goes together with a larger concentration of $\mathrm{CO} 2$. Because the damage function is assumed to be convex with the concentration of greenhouse gases, it also goes with larger damages, and with a larger societal benefit from early mitigation. This justifies a positive beta. We show in Section 7 that any credible calibration of a model combining the two sources of aggregate fluctuations yields a positive and large beta of mitigation. From our discussion above, this is compatible with using increasing discount rates to measure SCC. This provides a radical reversal in the trend of the literature on discounting. This suggests that rather than focusing on climate change, one should rather invest in negative-beta projects whose largest benefits materialize in the most catastrophic scenarii of the destiny of humankind on this planet.

This paper is organized as follows. In Section 2, we restate the classical pricing model with constant relative risk aversion and an arithmetic Brownian motion for the logarithm of aggregate consumption. We also introduce the CGF method in that section. The core of the paper is in sections 3 and 5, in which we explore asset prices in our two basic specifications, i.e., the Gaussian process with an uncertain trend and volatility, and the model with an uncertain probability of catastrophes. In Section 4, we extend the Gaussian specification of Section 3 to mean-reversion. We show how these results allow us to evaluate a broad class of risky projects with non-constant betas in Section 6. An application to climate change is presented in Section 7.

\footnotetext{
${ }^{6}$ The climate sensitivity is a physical parameter that measures the relationship between the concentration of greenhouse gases in the atmosphere and the average temperature of the earth.
} 


\section{The benchmark Gaussian model}

We evaluate a marginal investment project that reduces current consumption by some sure amount $\varepsilon$ and that generates a flow of benefits $\left(\varepsilon F_{1}, \varepsilon F_{2}, \ldots\right)$ in the future, which can be uncertain seen from today. Random variables $F_{t}, t=1,2, \ldots$, have known distribution functions. In order to evaluate the social desirability of such a project, we measure its impact on the intertemporal social welfare

$$
W=u\left(c_{0}\right)+\sum_{t=1} e^{-\delta t} E u\left(c_{t}\right)
$$

where $u$ is the increasing and concave utility function of the representative agent, $\delta$ is her rate of pure preference of the present, and $c_{t}$ is the consumption level of the representative agent at date $t$, with domain in $\mathbb{R}_{+}$. Because $\varepsilon$ is assumed to be small, implementing the project increases intertemporal social welfare if and only if

$$
-1+\sum_{t=1} E\left[\frac{e^{-\delta t} F_{t} u^{\prime}\left(c_{t}\right)}{u^{\prime}\left(c_{0}\right)}\right] \geq 0 .
$$

This can be rewritten as a standard NPV formula:

$$
-1+\sum_{t=1} e^{-\rho_{t}\left(F_{t}\right) t} E F_{t} \geq 0
$$

where $\rho_{t}\left(F_{t}\right)$ is the rate at which the expected cash flow occurring in $t$ years should be discounted. This discount rate is written as follows

$$
\rho_{t}\left(F_{t}\right)=\delta-\frac{1}{t} \ln \frac{E F_{t} u^{\prime}\left(c_{t}\right)}{u^{\prime}\left(c_{0}\right) E F_{t}}=r_{f t}+\pi_{t}\left(F_{t}\right)
$$

It is traditional in the CCAPM to decompose the project-specific discount rate $\rho_{t}\left(F_{t}\right)$ into a risk free discount rate $r_{f t}$ and a project-specific risk premium $\pi_{t}\left(F_{t}\right)$. From (4), we define these two components of the discount rate as follows:

$$
r_{f t}=\delta-\frac{1}{t} \ln \frac{E u^{\prime}\left(c_{t}\right)}{u^{\prime}\left(c_{0}\right)}
$$




$$
\pi_{t}\left(F_{t}\right)=-\frac{1}{t} \ln \frac{E F_{t} u^{\prime}\left(c_{t}\right)}{E F_{t} E u^{\prime}\left(c_{t}\right)}
$$

Observe that the risk premium $\pi_{t}(F)$ is zero when the project is safe or when its future cash flow is independent of future aggregate consumption. This implies that $r_{f t}$ is indeed the rate at which safe projects should be discounted. The CCAPM also characterizes the project-specific risk premium $\pi_{t}\left(F_{t}\right)$. Throughout the paper, we assume that $u^{\prime}(c)=c^{-\gamma}$ and that

$$
F_{t}=\xi_{t} c_{t}^{\beta}
$$

where $\left(\xi_{t}\right)_{t=1,2, \ldots}$ is a set of random variables independent of $c_{t}$, and $\beta$ is the CCAPM beta of the project (see for example Martin (2012)). Because the idiosyncratic risk $\xi_{t}$ is not priced, we hereafter identify a project $\left\{F_{t}\right\}$ by its $\beta$. When $\beta$ is positive, implementing the project raises the risk on aggregate consumption. When $\beta$ is negative, the project has an insurance component since it pays more on average in the worse macroeconomic scenarii.

Under this specification, asset pricing formulas (5) and (6) can be rewritten as follows:

$$
\begin{gathered}
r_{f t}=\delta-t^{-1} \chi\left(-\gamma, G_{t}\right), \\
\pi_{t}(\beta)=t^{-1}\left(\chi\left(\beta, G_{t}\right)+\chi\left(-\gamma, G_{t}\right)-\chi\left(\beta-\gamma, G_{t}\right)\right),
\end{gathered}
$$

where $G_{t}=\ln c_{t} / c_{0}$ is $\log$ consumption growth, and $\chi(a, x)=\ln E \exp (a x)$ is the CumulantGenerating Function (CGF) associated to random variable $x$ evaluated at $a \in \mathbb{R}$. CGF has recently been used by Martin (2012) to explore asset prices under non Gaussian economic growth processes. The CGF, if it exists, is the log of the better known moment-generating function. In this paper, we use the following properties of CGF (see Billingsley (1995)).

Lemma 1 : If it exists, the CGF function $\chi(a, x)=\ln E \exp (a x)$ has the following properties:

i. $\quad \chi(a, x)=\sum_{n=1}^{\infty} \kappa_{n}^{x} a^{n} / n$ ! where $\kappa_{n}^{x}$ is the $n$th cumulant of random variable $x$. If $m_{n}^{x}$ denotes the centered moment of $x$, we have that $\kappa_{1}^{x}=E x, \kappa_{2}^{x}=m_{2}^{x}, \kappa_{3}^{x}=\mu_{3}^{x}, \kappa_{4}^{x}=m_{4}^{x}-3\left(m_{2}^{x}\right)^{2}, \ldots$ 
ii. The most well-known special case is when $x$ is $N\left(\mu, \sigma^{2}\right)$, so that $\chi(a, x)=a \mu+0.5 a^{2} \sigma^{2}$.

iii. $\quad \chi(a, x+y)=\chi(a, x)+\chi(a, y)$ when $x$ and $y$ are independent random variables.

iv. $\quad \chi(0, x)=0$ and $\chi(a, x)$ is infinitely differentiable and convex in $a$.

$v$. This implies that $a^{-1} \chi(a, x)$ is increasing in $a$, from Ex to the supremum of the support of $x$ when a goes from zero to infinity.

vi. The cumulant of the $n$th order is homogeneous of order $n: \kappa_{n}^{\lambda x}=\lambda^{n} \kappa_{n}^{x}$ for all $\lambda \in \mathbb{R}$.

Property $i$ explains why $\chi$ is called the cumulant-generating function, and it links the sequence of cumulants to those of the centered moments. The first cumulant is the mean. The second, the third and the fourth cumulants are respectively the variance the skewness and the excess kurtosis of the random variable. Because the cumulants of the normal distribution are all zero for orders $n$ larger than 2, the CGF of a normally distributed $x$ is a quadratic function of $a$, as expressed by property ii. ${ }^{7}$ This property also implies that the CGF of a Dirac distribution degenerated at $x=x_{0} \in \mathbb{R}$ is equal to $a x_{0}$. Property $v$ will play a crucial role in this paper because of the assumption of an i.i.d. process for the growth of log consumption.

In the remainder of this paper, we calibrate equations (8) and (9) for different specifications of the stochastic process of $G_{t}$. The benchmark process is such that log consumption follows an arithmetic Brownian motion with trend $\mu$ and volatility $\sigma$. This implies that $G_{t}$ is normally distributed with mean $\mu t$ and variance $\sigma^{2} t$. Using property ii in Lemma 1 implies that equations (8) and (9) can be rewritten as follows

$$
r_{f t}=\delta+\gamma \mu-0.5 \gamma^{2} \sigma^{2}
$$

and

$$
\pi_{t}(\beta)=\beta \gamma \sigma^{2}=\beta \pi,
$$

Equation (10), which is often referred to as the extended Ramsey rule, holds independent of the maturity of the cash flow. In other words, the term structure of the safe discount rate is flat in that

\footnotetext{
${ }^{7}$ It should be noticed that the normal distribution is the only distribution that has a finite sequence of non-zero cumulants. This implies that the Gaussian case is the only one in which the equation in property $i$ in Lemma 1 can be used as an exact solution to the CGF.
} 
case. Its level is determined by three elements: impatience, a wealth effect and a precautionary effect. The wealth effect comes from the observation that investing for the future in a growing economy does increase intertemporal inequality. Because of inequality aversion (which is equivalent to risk aversion under the veil of ignorance), this is desirable only if the return of the project is large enough to compensate for this adverse effect on welfare. From (10), this wealth effect is equal to the product of the expected growth of log consumption by the degree $\gamma$ of concavity of the utility function which measures inequality aversion. The precautionary effect comes from the observation that consumers want to invest more for the future when this future is more uncertain (Drèze and Modigliani (1972), Kimball (1990)). This tends to reduce the discount rate. The precautionary effect is proportional to the volatility of the growth of log consumption.

Equation (11) tells us that the project-specific risk premium $\pi_{t}(\beta)$ is just equal to the product of the project-specific beta by the CCAPM aggregate risk premium $\pi=\gamma \sigma^{2}$. Under this standard specification, the risk premium associated to benefit $F_{t}$ is independent of its maturity $t$. The standard calibration of these two equations yields a too large risk free rate (risk free rate puzzle (Weil (1989))) and a too small risk premium (equity premium puzzle (Mehra and Prescott (1985))) compared to historical market data. Barro (2006) showed that these two puzzles can be solved by introducing a small probability of economic catastrophes in the stochastic growth process.

Because both the risk free rate and the risk premium of the project are independent of the maturity in this benchmark specification, their sum $\rho_{t}(\beta)=r_{f t}+\pi_{t}(\beta)$ is also independent of $t$. The term structure of risky discount rates is flat in this case. The risky discount rate equals

$$
\rho_{t}(\beta)=\delta+\gamma \mu+\gamma(\beta-0.5 \gamma) \sigma^{2}
$$

Notice that the risky discount rate can be either increasing or decreasing in the aggregate uncertainty measured by $\sigma^{2}$ depending upon whether the $\beta$ of the project is larger or smaller than $\gamma / 2$. Two competing effects are at play here. First, a large aggregate risk induces the representative agent to save more for the future (precautionary saving motive). That reduces the risk free discount rate. Second, ceteris paribus, a larger aggregate risk increases the projectspecific risk and the associated risk premium. This risk aversion effect is proportional to the beta 
of the project. The two effects counterbalance each other perfectly when $\beta=\gamma / 2$. When $\beta$ is smaller than $\gamma / 2$, the risk aversion effect (which is increasing in $\beta$ ) is dominated by the precautionary effect (which is increasing in $\gamma$ ).

\section{The Gaussian model with parametric uncertainty}

Following Weitzman (2007) and Gollier (2008), we now characterize the term structure of the risk free rate and the risk premium when there is some uncertainty about the true value of some of the parameters of the model. We assume in this section that $g_{t}=\ln \left(c_{t} / C_{t-1}\right)$ conditional to some unknown parameter $\theta$ follows a random walk. Since $G_{t}=\sum_{\tau=1}^{t} g_{\tau}$, we can rewrite equation (8) as follows:

$$
\begin{aligned}
r_{f t} & =\delta-t^{-1} \ln E\left[E e^{-\gamma \sum_{\tau}^{t} g_{\tau}} \mid \theta\right] \\
& =\delta-t^{-1} \ln E\left[\left(E e^{-\gamma g}\right)^{t} \mid \theta\right] \\
& =\delta-t^{-1} \ln E e^{t \chi(-\gamma, g \mid \theta)} \\
& =\delta-t^{-1} \chi(t, \chi(-\gamma, g \mid \theta)) .
\end{aligned}
$$

This means that the term structure of the risk free rate is determined by a sequence of two CGF operations. One must first compute $c(\theta)=\chi(-\gamma, g \mid \theta)$, which is the CGF of the per-period growth $g$ conditional to $\theta$. One must then compute $\chi(t, c)$ by using the distribution $P$ of $\theta$ that characterizes current beliefs. Notice that, by application of property $v$ of Lemma 1 , this immediately implies that the risk free discount rate has a decreasing term structure.

A similar exercise can be performed on equation (9). Using notation $g$ for $g \mid \theta$, this yields

$$
\pi_{t}(\beta)=t^{-1}(\chi(t, \chi(\beta, g))+\chi(t, \chi(-\gamma, g))-\chi(t, \chi(\beta-\gamma, g)))
$$

Observe that this equation implies that $\pi_{t}(0)=0$ for all $t$, and that, by application of property $v$ of Lemma $1, \pi_{t}(\beta)$ has an increasing term structure in the special case $\beta=\gamma$. 
We examine a special case of this model with parametric uncertainty by assuming that, conditional to $\theta$, the $\log$ of consumption growth is normally distributed with drift $\mu_{\theta}$ and volatility $\sigma_{\theta}$. Applying property ii of Lemma 1 to equation (13), we can write that

$$
\left.r_{f t}=\delta-t^{-1} \chi\left(t,-\gamma \mu+0.5 \gamma^{2} \sigma^{2}\right)\right)
$$

Similarly, we obtain that

$$
\pi_{t}(\beta)=t^{-1}\left(\chi\left(t, \beta \mu+0.5 \beta^{2} \sigma^{2}\right)+\chi\left(t,-\gamma \mu+0.5 \gamma^{2} \sigma^{2}\right)-\chi\left(t,(\beta-\gamma) \mu+0.5(\beta-\gamma)^{2} \sigma^{2}\right)\right) .
$$

Combining these two equations gives us the discount rate:

$$
\rho_{t}(\beta)=t^{-1}\left(\chi\left(t, \beta \mu+0.5 \beta^{2} \sigma^{2}\right)-\chi\left(t,(\beta-\gamma) \mu+0.5(\beta-\gamma)^{2} \sigma^{2}\right)\right)
$$

We apply these pricing formulas to different structures of uncertainty about the trend and the volatility of the growth of the economy. Property iii of Lemma 1 tells us that these two sources of uncertainty can be examined separately without loss of generality if they are independent. But before examining these two sources of uncertainty, let us observe that, since $\chi(a, x)=a E x+o\left(a^{2}\right)$, the above two equations imply that

$$
r_{f t}=\delta+\gamma E \mu-0.5 \gamma^{2} E \sigma^{2}+o(t)
$$

and

$$
\pi_{t}(\beta)=\beta \gamma E \sigma^{2}+o(t)
$$

Comparing these results with equations (10) and (11) yields the following result, which may explain why parametric uncertainty has not been much studied in asset pricing theory. The remainder of this section demonstrates that parametric uncertainty has more radical effects on prices of long-dated assets.

Proposition 1: In the Gaussian case, the parametric uncertainty affecting the trend and the volatility of economic growth has no effect on the risk free rate and on the risk premium for small maturities. 


\subsection{The trend of growth is uncertain}

Suppose first that log consumption follows an arithmetic Brownian motion with a known constant volatility $\sigma$ and an unknown constant trend $\mu_{\theta}$. Using properties $i$ and iii of Lemma 1 implies that equation (15) can then be rewritten as follows:

$$
r_{f t}=\delta-0.5 \gamma^{2} \sigma^{2}-t^{-1} \chi(t,-\gamma \mu)
$$

By properties $i$ and vi of Lemma 1, equation (20) also implies that

$$
r_{f t}=\delta-0.5 \gamma^{2} \sigma^{2}-\sum_{n=1}^{\infty} \frac{(-1)^{n} \gamma^{n} \kappa_{n}^{\mu} t^{n-1}}{n !}
$$

Let $m_{1}^{\mu}=E \mu$ and $m_{n}^{\mu}=E\left(\mu-m_{1}^{\mu}\right)^{n}$ for $n \geq 2$ denote respectively the mean and the centered moment of $\mu$. The above equation is thus equivalent to:

$$
r_{f t}=\delta+\gamma m_{1}^{\mu}-0.5 \gamma^{2} \sigma^{2}-\frac{1}{2 !} \gamma^{2} m_{2}^{\mu} t+\frac{1}{3 !} \gamma^{3} m_{3}^{\mu} t^{2}-\frac{1}{4 !} \gamma^{4}\left(m_{4}^{\mu}-3\left(m_{2}^{\mu}\right)^{2}\right) t^{3}+\ldots
$$

Two observations can be derived from this result. First, for small maturities, one more year in maturity implies a reduction of the discount rate by $0.5 \gamma^{2}$ times the variance of the trend $\mu$. If the trend of consumption growth is normally distributed, all terms involving higher cumulants of $\mu$ are zero, and the risk free discount rate is linearly decreasing with maturity, as shown by Gollier (2008). Second, if the distribution of the trend of growth is not normally distributed, the skewness $m_{3}^{\mu}$ and the excess kurtosis $m_{4}^{\mu}-3\left(m_{2}^{\mu}\right)^{2}$ also influence the shape of the term structure of $r_{f t}$ at larger maturities. A positive skewness or a negative excess kurtosis for the distribution of the trend $\mu$ tends to make the term structure increasing.

A similar exercise with equation (16) implies that

$$
\begin{aligned}
\pi_{t}(\beta) & =t^{-1}\left(\chi\left(t, \beta \mu+0.5 \beta^{2} \sigma^{2}\right)+\chi\left(t,-\gamma \mu+0.5 \gamma^{2} \sigma^{2}\right)-\chi\left(t,(\beta-\gamma) \mu+0.5(\beta-\gamma)^{2} \sigma^{2}\right)\right) \\
& =0.5 \sigma^{2}\left(\beta^{2}+\gamma^{2}-(\beta-\gamma)^{2}\right)+t^{-1}(\chi(t, \beta \mu)+\chi(t,-\gamma \mu)-\chi(t,(\beta-\gamma) \mu)) \\
& =\beta \gamma \sigma^{2}+t^{-1} \sum_{t=1}^{\infty} \frac{\kappa_{n}^{\mu} t^{n}}{n !}\left(\beta^{n}+(-\gamma)^{n}-(\beta-\gamma)^{n}\right) .
\end{aligned}
$$

This is equivalent to 


$$
\pi_{t}(\beta)=\beta \gamma\left(\sigma^{2}+m_{2}^{\mu} t\right)-0.5 \beta \gamma(\beta-\gamma) m_{3}^{\mu} t^{2}+\frac{1}{6} \beta \gamma\left(\beta^{2}-1.5 \beta \gamma+\gamma^{2}\right)\left(m_{4}^{\mu}-3\left(m_{2}^{\mu}\right)^{2}\right) t^{3}+\ldots
$$

Notice that for small maturities, the risk premium is increasing with $t$. One more year of maturity has an effect on the risk premium that is equivalent to an increase in the volatility of the log consumption growth $\sigma^{2}$ by the variance $m_{2}^{\mu}$ of the trend. If this trend is normally distributed, the other terms in the right-hand side of the above equation vanish, which implies that the risk premium is linearly increasing with maturity. For projects with a positive $\beta$ smaller than $\gamma$, a negative skewness reinforces the increasing nature of the term structure of $\pi_{t}(\beta)$ for positive betas, in particular for long maturities. A positive excess kurtosis has the same effect for projects with a positive beta. It must be noticed also that the risk premium is generally not proportional to $\beta$ as in the Gaussian CCAPM model. This implies that knowing the aggregate risk premium $\pi_{t}(1)$ and the beta of the project is not enough to determine the risk premium $\pi_{t}(\beta)$ associated to the project.

Let us now consider the discount rate $\rho_{t}(\beta)$ for a risky project. In the special case of a normally distributed trend, the above analysis implies that

$$
\rho_{t}(\beta)=\rho_{0}(\beta)+t \gamma m_{2}^{\mu}(\beta-0.5 \gamma)
$$

with $\rho_{0}(\beta)=\delta+\gamma m_{1}^{\mu}-0.5 \gamma^{2} \sigma^{2}+\beta \gamma \sigma^{2}$. We can summarize our findings relative to the shape of the term structures of $r_{f t}, \pi_{t}(\beta)$ and $\rho_{t}(\beta)=r_{f t}+\pi_{t}(\beta)$ in the following proposition.

Proposition 2: Suppose that log consumption follows an arithmetic Brownian motion with a known volatility $\sigma \in \mathbb{R}^{+}$and with an uncertain trend $\mu$ which is normally distributed. It implies that the term structures of the risk free discount rate and of the aggregate risk premium are respectively decreasing and increasing. Moreover, the term structure of the discount rates $\rho_{t}(\beta)$ is decreasing, flat or increasing depending upon $\beta$ is smaller, equal or larger than $0.5 \gamma$. These results hold in the non-Gaussian growth, but only for small maturities, or when the uncertainty on growth is such that the cumulants of orders larger than 2 are small compared to the variance. 
The intuition of these results combines the observation that the parametric uncertainty magnifies long term risks with the observation made in the previous section that risk decreases or increases the discount rate depending upon whether $\beta$ is smaller or larger than $\gamma / 2$.

In the next proposition, we generalize these findings to non-Gaussian distributions of the trend of growth. It is easy to generalize equation (25) to the case of a non-Gaussian $\mu$ :

$$
\rho_{t}(\beta)=\rho_{0}(\beta)+t^{-1} \chi\left(t, \beta\left(\mu-m_{1}^{\mu}\right)\right)-t^{-1} \chi\left(t,(\beta-\gamma)\left(\mu-m_{1}^{\mu}\right)\right)
$$

Notice that property $v$ of Lemma 1 applied to this equation implies that $\rho_{t}(\beta=0)$ and $\rho_{t}(\beta=\gamma)$ are respectively decreasing and increasing in $t$. And if $\mu$ has a symmetric distribution, the above equation implies that $\rho_{t}(\beta=0.5 \gamma)$ is constant in $t$ at $\rho_{0}(\gamma / 2)=\delta+\gamma m_{1}^{\mu}$. Under this symmetry assumption, we obtain the following result.

Proposition 3: Suppose that log consumption follows an arithmetic Brownian motion with a known volatility $\sigma \in \mathbb{R}^{+}$and with an unknown trend $\mu$. When $\mu$ is symmetrically distributed, the term structure of the discount rate $\rho_{t}(\beta=0.5 \gamma)$ is flat at $\delta+\gamma m_{1}^{\mu}$. It is decreasing when $\beta$ is smaller than $0.5 \gamma$, and it is increasing when $\beta$ is in interval $[0.5 \gamma, \gamma]$.

Proof: See the Appendix.

Thus, we recover essentially the same characterization for the slope of the term structure of $\rho_{t}(\beta)$ if we replace the Gaussian assumption contained in Proposition 2 by the assumption that this uncertainty is just symmetric around the mean.

In the next proposition, we characterize the asymptotic properties of the term structure of discount rates when the distribution of the trend has a bounded support $\left[\mu_{\min }, \mu_{\max }\right]$. We first rewrite condition (26) as

$$
\rho_{t}(\beta)=\delta+\gamma \sigma^{2}(\beta-0.5 \gamma)+t^{-1} \chi(t, \beta \mu)-t^{-1} \chi(t,(\beta-\gamma) \mu)
$$

Property $v$ of Lemma 1 tells us that $a^{-1} \chi(a, x)$ tends to the supremum of the support of $x$ when $a$ tends to infinity. If $\beta$ is negative, the supremum of the support of $\beta \mu$ is $\beta \mu_{\min }$, and the supremum 
of the support of $(\beta-\gamma) \mu$ is $(\beta-\gamma) \mu_{\min }$. This implies that the sum of the last two terms of the RHS of the above equality tends to $\gamma \mu_{\text {min }}$. The other two cases are characterized in Proposition 4.

Proposition 4: Suppose that log consumption follows an arithmetic Brownian motion with a known volatility $\sigma \in \mathbb{R}^{+}$and with an unknown trend $\mu$ with support $\left[\mu_{\min }, \mu_{\max }\right]$. For long horizons, the discount rate tends to

$$
\rho_{\infty}(\beta)=\left\{\begin{array}{llc}
\delta+\gamma \sigma^{2}(\beta-0.5 \gamma)+\gamma \mu_{\min } & \text { if } & \beta \leq 0 \\
\delta+\gamma \sigma^{2}(\beta-0.5 \gamma)+(\gamma-\beta) \mu_{\min }+\beta \mu_{\max } & \text { if } & 0<\beta \leq \gamma \\
\delta+\gamma \sigma^{2}(\beta-0.5 \gamma)+\gamma \mu_{\max } & \text { if } & \beta>\gamma
\end{array}\right.
$$

For distant futures, the ambiguity affecting the trend is crucial for the determination of the discount rate. The long term wealth effect is equal to the product of $\gamma$ by a growth rate of consumption belonging to its support $\left[\mu_{\min }, \mu_{\max }\right]$. Its selection depends here upon the beta of the project. When $\beta$ is negative, the wealth effect should be computed on the basis of the smallest possible growth rate $\mu_{\min }$ of the economy. On the contrary, when $\beta$ is larger than $\gamma$, the wealth effect should be computed on the basis of the largest possible rate $\mu_{\max }$. When the beta of the project is positive but smaller than $\gamma$, the selected growth rate is a weighted average of $\mu_{\min }$ and $\mu_{\max }$, with weights $(\gamma-\beta) / \gamma$ and $\beta / \gamma$ respectively.

Proposition 4 also tells us that the condition of a symmetric distribution for $\mu$ in Proposition 3 cannot be relaxed. Indeed, Proposition 4 implies that $\rho_{0}(\beta=\gamma / 2)$ and $\rho_{\infty}(\beta=\gamma / 2)$ are equal only if $E \mu=m_{1}^{\mu}$ and $\left(\mu_{\min }+\mu_{\max }\right) / 2$ coincide. Most asymmetric distributions will not satisfy this condition, which implies that the constancy of $\rho_{t}(\beta=\gamma / 2)$ with respect to $t$ will be violated.

In Figure 1, we illustrate some of the above findings through the following numerical example. We assume that $\delta=0, \gamma=2, \sigma=4 \%$ and $\mu$ is uniformly distributed on interval [0\%,3\%]. The term structure is flat for $\beta=\gamma / 2=1$. The sensitiveness of the discount rate for different betas to changes in the beta is increasing in the maturity of the cash flows. This numerical example also illustrates the property that project-specific risk premia are in general not proportional to the project-specific beta. For example, consider a time horizon of 400 years. For this maturity, the 
risk premia associated to $\beta=1$ and $\beta=4$ are respectively equal to $\pi_{400}(1)=2.5 \%$ and $\pi_{400}(4)=6.3 \%<4 \pi_{400}(1)$.
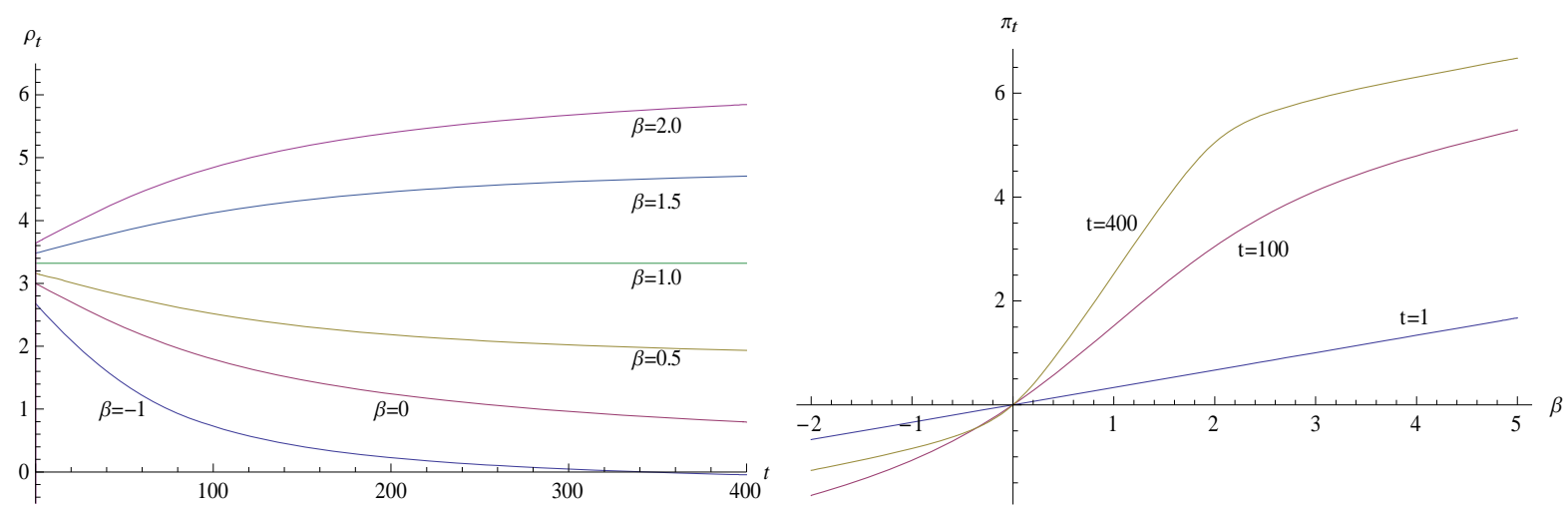

Figure 1: The discount rate as a function of maturity (left) and of the beta of the project (right). We assume that $\gamma=2, \sigma=4 \%$ and $\mu$ is $U[0 \%, 3 \%]$. Rates are in \%.

\subsection{The volatility of growth is uncertain}

Let us now turn to the case in which the trend is known, but the volatility is ambiguous. Weitzman (2007) examined this question by assuming that the $\sigma^{2}$ has an inverted Gamma distribution. This implies that $\ln \left(c_{t} / c_{0}\right)$ is a Student's t-distribution rather than a normal, yielding fat tails, a safe discount rate of $-\infty$ and a market risk premium of $+\infty$. Let us reexamine this question without specifying the distribution of $\sigma^{2}$. Lemma 1 implies that we can rewrite equation (15) as follows:

$$
\left.r_{f t}=\delta+\gamma \mu-t^{-1} \chi\left(t, 0.5 \gamma^{2} \sigma^{2}\right)\right)
$$

Because the Inverse Gamma distribution for $\sigma^{2}$ has no real CGF, this equation offers another proof of Weitzman (2007)'s inexistence result. Property $i$ of Lemma 1 also implies that

$$
\begin{aligned}
r_{f t} & =\delta+\gamma \mu-\sum_{n=1}^{\infty} \frac{\kappa_{n}^{0.5 \gamma^{2} \sigma^{2}} t^{n-1}}{n !} \\
& =\delta+\gamma \mu-0.5 \gamma^{2} m_{1}^{\sigma^{2}}-\frac{1}{8} \gamma^{4} m_{2}^{\sigma^{2}} t-\frac{1}{48} \gamma^{6} m_{3}^{\sigma^{2}} t^{2}-\frac{1}{384} \gamma^{8}\left(m_{4}^{\sigma^{2}}-3\left(m_{2}^{\sigma^{2}}\right)^{2}\right) t^{3}-\ldots
\end{aligned}
$$


This equation is useful to measure the impact of each cumulant of the distribution of $\sigma^{2}$ on the shape of the term structure of the risk free rate. Similarly, equation (16) implies that

$$
\pi_{t}(\beta)=t^{-1}\left(\chi\left(t, 0.5 \beta^{2} \sigma^{2}\right)+\chi\left(t, 0.5 \gamma^{2} \sigma^{2}\right)-\chi\left(t, 0.5(\beta-\gamma)^{2} \sigma^{2}\right)\right) .
$$

Lemma 1 implies in turn that

$$
\begin{aligned}
\pi_{t}(\beta) & =\sum_{t=1}^{\infty} \frac{\kappa_{n}^{\sigma^{2}} t^{n-1}}{2^{n} n !}\left(\beta^{2 n}+\gamma^{2 n}-(\beta-\gamma)^{2 n}\right) \\
& =\beta \gamma m_{1}^{\sigma^{2}}+\frac{1}{2} \beta \gamma m_{2}^{\sigma^{2}}\left[\beta^{2}-\frac{3}{2} \beta \gamma+\gamma^{2}\right] t+\ldots
\end{aligned}
$$

The bracketed term in the right-hand side of this equation is positive, which implies that the term structure of the aggregate risk premium is upward-sloping for small maturities. For longer maturities, the higher cumulants matter for the shape of the term structure of $\pi_{t}(\beta)$, the direction of the effect of cumulant $n$ depending upon the sign of $\beta^{2 n}+\gamma^{2 n}-(\beta-\gamma)^{2 n}$.

Finally, combining these two results implies that the discount rate $\rho_{t}(\beta)$ to be used for this project is such that

$$
\begin{aligned}
\frac{\partial \rho_{t}(\beta)}{\partial t} & =\frac{1}{8}\left[\beta^{4}-(\beta-\gamma)^{4}\right] m_{2}^{\sigma^{2}}+o(t) \\
& =\frac{\gamma(2 \beta-\gamma)\left(\beta^{2}+(\beta-\gamma)^{2}\right)}{8} m_{2}^{\sigma^{2}}+o(t) .
\end{aligned}
$$

Thus, for short maturities, the term structure of the discount rate to be used for risky projects is downward-sloping if and only if $\beta$ is smaller than $\gamma / 2$. This result is identical to what we obtained when the uncertainty is about the trend of the economy. The following proposition summarizes the findings of this section.

Proposition 5: Suppose that log consumption follows an arithmetic Brownian motion with a known trend $\mu$ and an uncertain volatility $\sigma$. It implies that the term structures of the risk free discount rate and of the aggregate risk premium are respectively decreasing and increasing for short maturities. Moreover, the term structure of the discount rates $\rho_{t}(\beta)$ is decreasing, flat or increasing for short maturities depending upon $\beta$ is smaller, equal or larger than $0.5 \gamma$. 
Given the similarities of these results with those of Proposition 2, we can conclude that, for small maturities, the qualitative properties of the term structures of discount rates are independent of whether the uncertainty is about the trend of growth or about its volatility.

\subsection{The trend and the volatility of growth are uncertain}

Many of the above results suggest that that the term structure of $\rho_{t}(\beta)$ is decreasing under small parametric uncertainty if and only if $\beta$ is smaller than $\gamma / 2$. This is not true in general, as shown

in this section. To do this, let us suppose that $\mu$ and $\sigma^{2}$ be statistically dependent. Applying property $i$ of Lemma 1 in equation (17) implies that

$$
\frac{\partial \rho_{t}(\beta)}{\partial t}=\frac{1}{2 !}\left(\kappa_{2}^{\beta \mu+0.5 \beta^{2} \sigma^{2}}-\kappa_{2}^{(\beta-\gamma) \mu+0.5(\beta-\gamma)^{2} \sigma^{2}}\right)+o(t)
$$

This implies that

$$
\begin{aligned}
2 \frac{\partial \rho_{t}(\beta)}{\partial t} & =\left[\beta^{2}-(\beta-\gamma)^{2}\right] m_{2}^{\mu}+0.25\left[\beta^{4}-(\beta-\gamma)^{4}\right] m_{2}^{\sigma^{2}} \\
& +\left[\beta^{3}-(\beta-\gamma)^{3}\right] \operatorname{cov}\left(\mu, \sigma^{2}\right)+o(t)
\end{aligned}
$$

In the special case with $\beta=\gamma / 2$, this implies in turn that

$$
\frac{\partial \rho_{t}(\beta)}{\partial t}=\frac{\gamma^{3}}{8} \operatorname{cov}\left(\mu, \sigma^{2}\right)+o(t)
$$

Thus, when $\beta=\gamma / 2$, the signs of the slope of the term structure of the discount rate for small maturities and of $\operatorname{cov}\left(\mu, \sigma^{2}\right)$ coincide. To illustrate this result, let us consider the following simple numerical example. Suppose that $\delta=0, \gamma=2$ and that there are two possible states of nature. In the first state, $\mu_{1}=0 \%$ and $\sigma_{1}=1 \%$. In the second state, $\mu_{2}=3 \%$ and $\sigma_{2}=7 \%$, so that the trend and the volatility of growth are positively correlated. Under this calibration of the model, the discount rate for short-term cash flows with $\beta=1=\gamma / 2$ is $\rho_{0}(1)=3 \%$, and the discount rate for distant cash flows is $\rho_{\infty}(1)=3.24 \%>\rho_{0}(1)$. 


\section{Extension to mean-reversion}

In the benchmark specification with a CRRA utility function and a Brownian motion for the growth of log consumption, the term structures of discount rates are flat and constant through time. In the specification examined in Section 3 with some parametric uncertainty on this Brownian motion, they are monotone and move smoothly through time due to the revision of beliefs about the true values of the uncertain parameters. But these processes ignore the cyclicality of the economic activity. The introduction of predictable changes in the trend of growth introduces a new ingredient to the evaluation of investments. When expectations are diminishing, the discount rate associated to short horizons should be reduced to bias investment decisions toward projects that dampen the forthcoming recession. Long termism is a luxury that should be favored only in periods of economic prosperity with low expectations for the future. More generally, when expectations are cyclical, it is important to frequently adapt the price signals contained in the term structure of discount rates to the moving macroeconomic expectations. From this theoretical result, it is clearly inefficient to maintain the U.S. official discount rate unchanged since 1992.

In this section, we propose a simple model in which the economic growth is cyclical, with some uncertainty about the parameter governing this process. Following Bansal and Yaron (2004) for example, the change in log consumption follows an auto-regressive process:

$$
\begin{aligned}
& \ln c_{t+1} / c_{t}=x_{t} \\
& x_{t}=\mu_{\theta}+y_{t}+\varepsilon_{x t}, \\
& y_{t}=\phi y_{t-1}+\varepsilon_{y t},
\end{aligned}
$$

for some initial (potentially ambiguous) state characterized by $y_{-1}$, where $\varepsilon_{x t}$ and $\varepsilon_{y t}$ are independent and serially independent with mean zero and variance $\sigma_{x}^{2}$ and $\sigma_{y}^{2}$, respectively. Parameter $\phi$, which is between 0 and 1 , represents the degree of persistence in the expected growth rate process. When $\phi$ is zero, then the model returns to a pure random walk as in Section 
3. We hereafter allow the trend of growth $\mu_{\theta}$ to be uncertain. ${ }^{8}$ By forward induction of (37), it follows that:

$$
G_{t}=\ln c_{t} / c_{0}=\mu_{\theta} t+y_{-1} \phi \frac{1-\phi^{t}}{1-\phi}+\sum_{\tau=0}^{t-1} \frac{1-\phi^{t-\tau}}{1-\phi} \varepsilon_{y \tau}+\sum_{\tau=0}^{t-1} \varepsilon_{x \tau}
$$

It implies that, conditional to $\theta, G_{t}$ is normally distributed with annualized variance

$$
t^{-1} \operatorname{Var}\left(\ln c_{t} / c_{0}\right)=\frac{\sigma_{y}^{2}}{(1-\phi)^{2}}\left[1-2 \phi \frac{\phi^{t}-1}{t(\phi-1)}+\phi^{2} \frac{\phi^{2 t}-1}{t\left(\phi^{2}-1\right)}\right]+\sigma_{x}^{2}
$$

Using property ii of Lemma 1, equations (8) and (9) imply that

$$
\begin{aligned}
\rho_{t}(\beta)= & t^{-1}\left(\chi\left(\beta, G_{t}\right)-\chi\left(\beta-\gamma, G_{t}\right)\right) \\
=\delta+ & \gamma y_{-1} \phi \frac{1-\phi^{t}}{t(1-\phi)}+\gamma(\beta-0.5 \gamma)\left(\frac{\sigma_{y}^{2}}{(1-\phi)^{2}}\left[1-2 \phi \frac{\phi^{t}-1}{t(\phi-1)}+\phi^{2} \frac{\phi^{2 t}-1}{t\left(\phi^{2}-1\right)}\right]+\sigma_{x}^{2}\right) \\
& \quad+t^{-1}(\chi(t, \beta \mu)-\chi(t,(\beta-\gamma) \mu))
\end{aligned}
$$

One can then treat the last term in the RHS of this equation as in Section 3.1. Bansal and Yaron (2004) consider the following calibration of the model, using annual growth data for the United States over the period 1929-1998. Taking the month as the time unit, they obtained, $\mu=0.0015$, $\sigma_{x}=0.0078, \sigma_{y}=0.00034$, and $\phi=0.979$. Using this $\phi$ yields a half-life for macroeconomic shocks of 32 months. Let us assume that $\delta=0$, and let us introduce some uncertainty about the historical trend of growth from the sure $\mu=0.0015$ to the uncertain context with two equally likely trends $\mu_{1}=0.0005$ and $\mu_{2}=0.0025$. In Figure 2, we draw the term structures of discount rates for two different positions $y_{-1}$ in the business cycle. In the left figure, the expected annual growth rate of the economy is $0.6 \%$ per year, well below its unconditional expectation of $1.8 \%$. In this recession phase, the short term discount rate is a low $1 \%$, but the expectation of a recovery makes the term structure steeply increasing for low maturities. For betas below unity, the term structure is non-monotone because of the fact that for very distant maturities, the effect of parametric uncertainty eventually dominates. In the right figure, the expected instantaneous

\footnotetext{
${ }^{8}$ A more general model entails a time-varying volatility of growth as in Bansal and Yaron (2004). Mean-reversion in volatility is useful to explain the cyclicality of the market risk premium.
} 
growth rate is $1.2 \%$ per year above its unconditional expectation. In this expansionary phase of the cycle, the short term discount rate is large at around 6\%, but is steeply decreasing for short maturities because of the diminishing expectations.
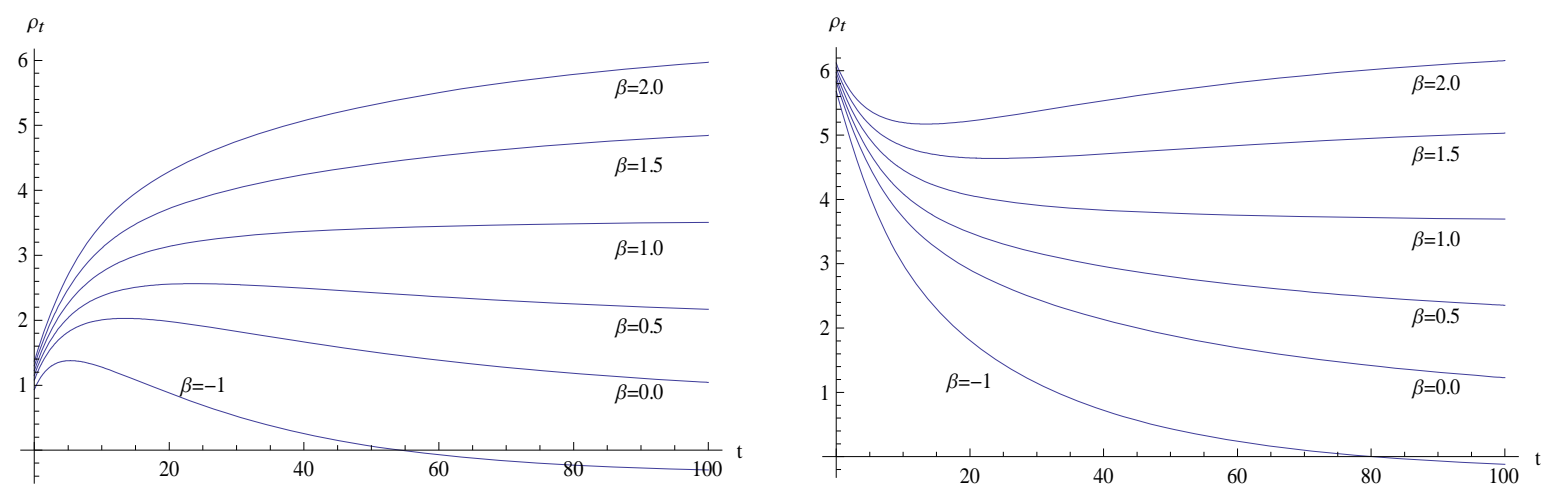

Figure 2: The discount rate (in \% per year) as a function of the maturity (in years) in recession for different betas. Equation (40) is calibrated with $\delta=0, \gamma=2, \sigma_{x}=0.0078, \sigma_{y}=0.00034$, $\phi=0.979$, two equally likely trends $\mu_{1}=0.0005$ and $\mu_{2}=0.0025$, and $y_{-1}=-0.001$ (left) or for $y_{-1}=0.001$ (right).

One can also examine a model in which the current state variable $y_{-1}$ is uncertain. It is easy to generalize equation (40) to examine this ambiguous context. We obtain the following pricing formula:

$$
\begin{aligned}
& \rho_{t}(\beta)=\delta+\gamma(\beta-0.5 \gamma)\left(\frac{\sigma_{y}^{2}}{(1-\phi)^{2}}\left[1-2 \phi \frac{\phi^{t}-1}{t(\phi-1)}+\phi^{2} \frac{\phi^{2 t}-1}{t\left(\phi^{2}-1\right)}\right]+\sigma_{x}^{2}\right) \\
& +t^{-1}\left(\chi\left(t, \beta \mu+\beta y \phi\left(1-\phi^{t}\right) / t(1-\phi)\right)-\chi\left(t,(\beta-\gamma) \mu+(\beta-\gamma) y \phi\left(1-\phi^{t}\right) / t(1-\phi)\right)\right) .
\end{aligned}
$$

Observe again that when $\beta=0.5 \gamma$, the term structure of discount rates is flat if both $\mu$ and $y_{-1}$ have a symmetric distribution function. We also observe that the ambiguity on $y_{-1}$ plays a role similar to the ambiguity on $\mu$ to shape the term structure. Our numerical simulations (available upon request) show that the hidden nature of the state variable does not modify the general characteristics of the term structures described above.

\section{A model with rare events of unknown frequency}


In a recent move of the literature initiated by Barro (2006, 2009), and followed for example by Backus, Chernov and Martin (2011) and Martin (2012), rare events have been recognized for being a crucial determinant of assets prices. The underlying model is such that the growth of log consumption $g_{t}$ follows an i.i.d. process with a CRRA utility function, so that equations (13), (14) and (17) are relevant. However, contrary to what has been assumed in the Section 3, $g_{t}=g$ is not normally distributed conditional to $\theta$. Rather, it compounds two normal distributions:

$$
g \sim\left(h_{1}, 1-p ; h_{2}, p\right) \quad \text { with } \quad h_{i} \sim N\left(\mu_{i}, \sigma_{i}^{2}\right)
$$

The log consumption growth compounds a "business-as-usual” random variable $h_{1} \sim N\left(\mu_{1}, \sigma_{1}^{2}\right)$ with probability $1-p$, with a catastrophe event $h_{2} \sim N\left(\mu_{2}, \sigma_{2}^{2}\right)$ with probability $p$ and $\mu_{2}<<0<\mu_{1}$ and $\sigma_{2} \geq \sigma_{1}$. Barro $(2006,2009)$ convincingly explains that the risk free puzzle and the equity premium puzzle can be explained by using credible values of the intensity $\mu_{2}$ of the macro catastrophe and of its frequency $p$. However, Martin (2012) shows that the levels of the $r_{f}$ and $\pi(1)$ are highly sensitive to the frequency $p$, and that this parameter $p$ is extremely difficult to estimate. In this section, we contribute to this emerging literature by integrating this source of parametric uncertainty into the asset pricing model.

In the absence of parametric uncertainty, equations (13), (14) and (17) imply that the term structures of discount rates and risk premiums are flat. Suppose alternatively that parameter $p$ is uncertain. Our current beliefs about the true frequency of macro catastrophes are given by some probability distribution $P$ on $p$. Let $\left[p_{\min }, p_{\max }\right]$ denote the support of $P$. In this section, we calibrate this model as in the EU benchmark version of Barro (2006) and Martin (2012). We assume that $\delta=3 \%, \gamma=4$, and $n=2$. In the business-as-usual scenario, the trend of growth is $\mu_{1}=2.5 \%$ and its volatility is equal to $\sigma_{1}=2 \%$. In case of a catastrophe, the trend of growth is $\mu_{2}=-39 \%$ and the volatility is $\sigma_{2}=25 \%$. Finally, we assume that the probability of catastrophe is $1.2 \%$ or $2.2 \%$ with equal probabilities. ${ }^{9}$

At this stage, it is useful to define the following set of parameters and functions:

\footnotetext{
${ }^{9}$ This corresponds to the two sensitivity analyses performed by Martin (2012) around Barro’s estimation of $p=1.7 \%$.
} 


$$
\begin{aligned}
& a_{i}(\beta)=\exp \left(\beta \mu_{i}+0.5 \beta^{2} \sigma_{i}^{2}\right) \\
& b_{i}=\exp \left(-\gamma \mu_{i}+0.5 \gamma^{2} \sigma_{i}^{2}\right) \\
& d_{i}(\beta)=\exp \left((\beta-\gamma) \mu_{i}+0.5(\beta-\gamma)^{2} \sigma_{i}^{2}\right)
\end{aligned}
$$

for $i=1,2$. Remember that these variables represent respectively the expectation of $F_{1}$, $u^{\prime}\left(c_{1}\right) / u^{\prime}\left(c_{0}\right)$ and $F_{1} u^{\prime}\left(c_{1}\right) / u^{\prime}\left(c_{0}\right)$ conditional to scenario $i$.

In this context, equation (13) becomes

$$
r_{f t}=\delta-t^{-1} \chi\left(t, \ln \left(b_{1}+p\left(b_{2}-b_{1}\right)\right)\right)
$$

Using Lemma 1, we directly obtain the following results. First, the term structure of $r_{f t}$ is decreasing. This means that rare events are inherently linked to a decreasing structure of the risk free rate because of the intrinsic uncertainty related to their low frequency. For small maturities, it tends to

$$
r_{f 0}=\delta-E \ln \left(b_{1}+p\left(b_{2}-b_{1}\right)\right)
$$

By Jensen's inequality, $r_{f 0}$ is larger than if we would have assumed a sure frequency equaling Ep. This implies that the uncertainty affecting the frequency of rare events raises the risk free discount rate for short maturities. However, under our calibration, this effect is small. It raises the discount rate from $0.46 \%$ to $0.52 \%$ when replacing a sure frequency of $1.7 \%$ to an uncertain frequency $p \sim(1.2 \%, 1 / 2 ; 2.2 \%, 1 / 2)$.

It would however be a mistake to conclude from this analysis that introducing uncertainty in Barro's model has a negligible effect on the discount rate. Property $i$ in Lemma 1 tells us that one can rewrite equation (44) has follows:

$$
r_{f t}=r_{f 0}-\sum_{n=2}^{\infty} \frac{\kappa_{n}^{\ln \left(b_{1}+p\left(b_{2}-b_{1}\right)\right)} t^{n-1}}{n !} .
$$

This implies that

$$
\frac{\partial r_{f t}}{\partial t}=-0.5 m_{2}^{\ln \left(b_{1}+p\left(b_{2}-b_{1}\right)\right)}+o(t) .
$$


In the calibration of this section, we have that the first term in the RHS of this equation is equal to $-0.06 \%$ per year, which is quite large. Lemma 1 can also be used to determine the asymptotic risk free discount rate. Because $b_{2}>b_{1}$ under risk aversion, it yields

$$
\lim _{t \rightarrow \infty} r_{f t}=\delta-\ln \left(b_{1}+p_{\max }\left(b_{2}-b_{1}\right)\right)
$$

That is, the long discount rate should be computed by using the CCAPM pricing equation under the belief that the largest possible frequency of catastrophes is certain. This pessimistic approach valuation is compatible with a smaller discount rate for the distant future. Under our calibration, we obtain $r_{f \infty}=-2.86 \%$, a rate that should be compared to rate $r_{f \infty}=0.46 \%$ that holds in the absence of parametric uncertainty. A full description of the term structure of $r_{f t}$ is given in Figure 3.

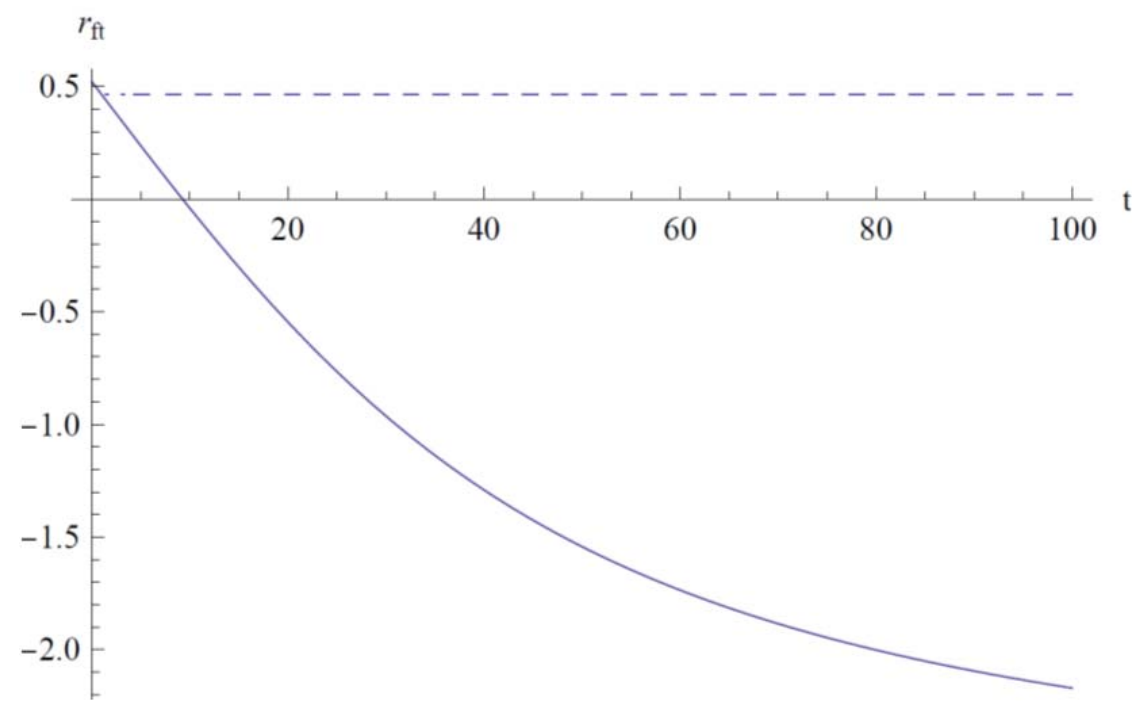

Figure 3: The term structure of risk free discount rates (in \%) calibrated with $\delta=3 \%, \gamma=4$, $n=2, \mu_{1}=2.5 \%, \sigma_{1}=2 \%, \mu_{2}=-39 \%$ and $\sigma_{2}=25 \%$. In the unambiguous case (dashed), the probability $p$ equals $1.7 \%$ with certainty. In the ambiguous case (plain), it is $1.2 \%$ or $2.2 \%$ with equal probabilities.

Let us now turn to the risk premium. Using specification (42) in equation (14), we obtain

$$
\begin{aligned}
\pi_{t}(\beta)= & t^{-1} \chi\left(t, \ln \left[a_{1}(\beta)+p\left(a_{2}(\beta)-a_{1}(\beta)\right)\right]\right)+t^{-1} \chi\left(t, \ln \left[b_{1}+p\left(b_{2}-b_{1}\right)\right]\right) \\
& -t^{-1} \chi\left(t, \ln \left[d_{1}(\beta)+p\left(d_{2}(\beta)-d_{1}(\beta)\right)\right]\right) .
\end{aligned}
$$

For short maturities, we obtain that 


$$
\pi_{0}(\beta)=E \ln \frac{\left[a_{1}(\beta)+p\left(a_{2}(\beta)-a_{1}(\beta)\right)\right]\left[b_{1}+p\left(b_{2}-b_{1}\right)\right]}{d_{1}(\beta)+p\left(d_{2}(\beta)-d_{1}(\beta)\right)} .
$$

For longer maturities, we can use property $i$ of Lemma 1 to obtain

$$
\pi_{t}(\beta)=\pi_{0}(\beta)+\sum_{n=2}^{\infty} \frac{t^{n-1}}{n !}\left(\kappa_{n}^{\ln \left(a_{1}(\beta)+p\left(a_{2}(\beta)-a_{1}(\beta)\right)\right)}+\kappa_{n}^{\ln \left(b_{1}+p\left(b_{2}-b_{1}\right)\right)}-\kappa_{n}^{\ln \left(d_{1}(\beta)+p\left(d_{2}(\beta)-d_{1}(\beta)\right)\right)}\right) .
$$

This implies that

$$
\frac{\partial \pi_{t}(\beta)}{\partial t}=0.5\left(m_{2}^{\ln \left(a_{1}(\beta)+p\left(a_{2}(\beta)-a_{1}(\beta)\right)\right)}+m_{2}^{\ln \left(b_{1}+p\left(b_{2}-b_{1}\right)\right)}-m_{2}^{\ln \left(d_{1}(\beta)+p\left(d_{2}(\beta)-d_{1}(\beta)\right)\right)}\right)+o(t)
$$

In the calibration of this section, we obtain that the slope of the term structure of the risk premium at small maturities is equal to respectively $0.04 \%$ and $0.06 \%$ respectively for $\beta=1$ and $\beta=5$.

The asymptotic value of the risk premium equals

$$
\begin{aligned}
\lim _{t \rightarrow \infty} \pi_{t}(\beta) & =\sup \ln \left(a_{1}(\beta)+p\left(a_{2}(\beta)-a_{1}(\beta)\right)\right)+\sup \ln \left(b_{1}+p\left(b_{2}-b_{1}\right)\right) \\
& -\sup \ln \left(d_{1}(\beta)+p\left(d_{2}(\beta)-d_{1}(\beta)\right)\right) .
\end{aligned}
$$

It is easy to show that $a_{2}(\beta)<a_{1}(\beta)$ if and only if $\beta$ is positive and smaller than $\alpha=2\left(\mu_{1}-\mu_{2}\right) /\left(\sigma_{1}^{2}-\sigma_{2}^{2}\right)$. Similarly, $d_{2}(\beta)<d_{1}(\beta)$ if and only if $\beta$ is between $\gamma$ and $\gamma+\alpha$. This implies for example that

$$
\lim _{t \rightarrow \infty} \pi_{t}(\beta)=\ln \frac{\left(a_{1}(\beta)+p_{\text {min }}\left(a_{2}(\beta)-a_{1}(\beta)\right)\right)\left(b_{1}+p_{\text {max }}\left(b_{2}-b_{1}\right)\right)}{d_{1}(\beta)+p_{\text {max }}\left(d_{2}(\beta)-d_{1}(\beta)\right)},
$$

when $\beta$ belongs to interval $[0, \min \{\gamma, \alpha\}]$. In the numerical example presented above, we have that $\gamma=4$ and $\alpha=13.4$. This means that condition (54) holds for a wide range of projects and assets with $\beta \in[0,4]$. In Figure 4, we draw the term structure of the risk premium $\pi_{t}(\beta)$ for $\beta=1$. It increases from $\pi_{0}(1)=5.87 \%$ to $\pi_{\infty}(1)=7.71 \%$. 


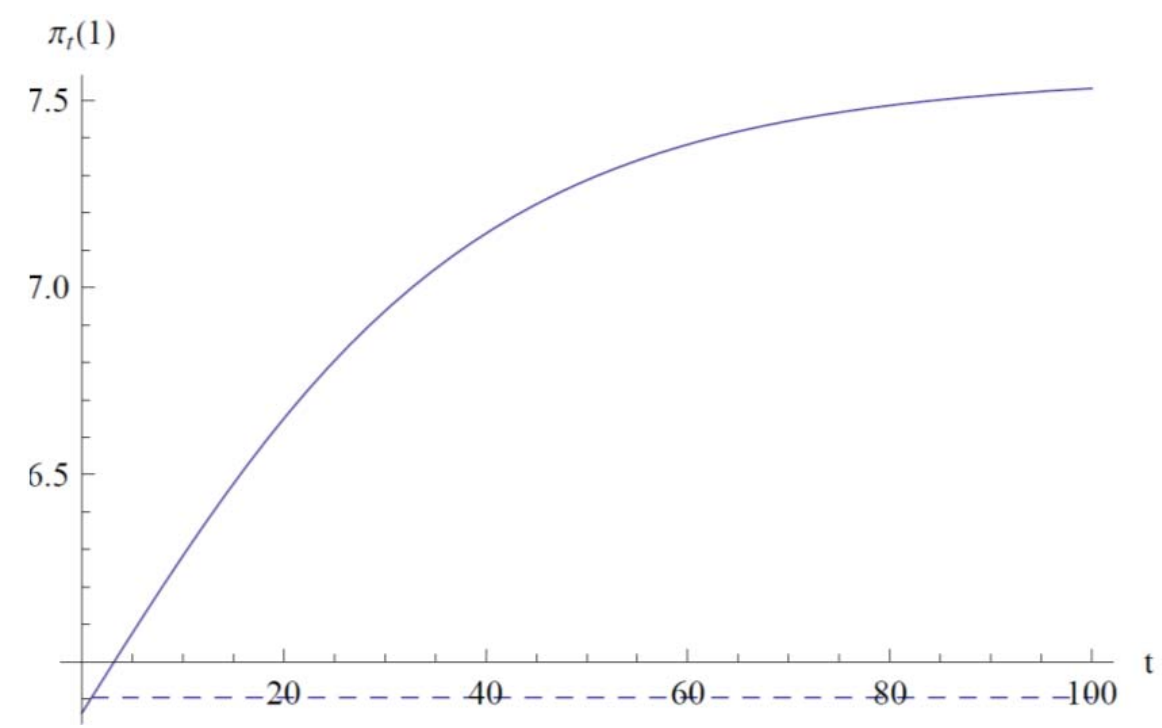

Figure 4: The term structure of the risk premium $\pi_{t}(1)$ (in \%). The calibration is as in Figure 3.

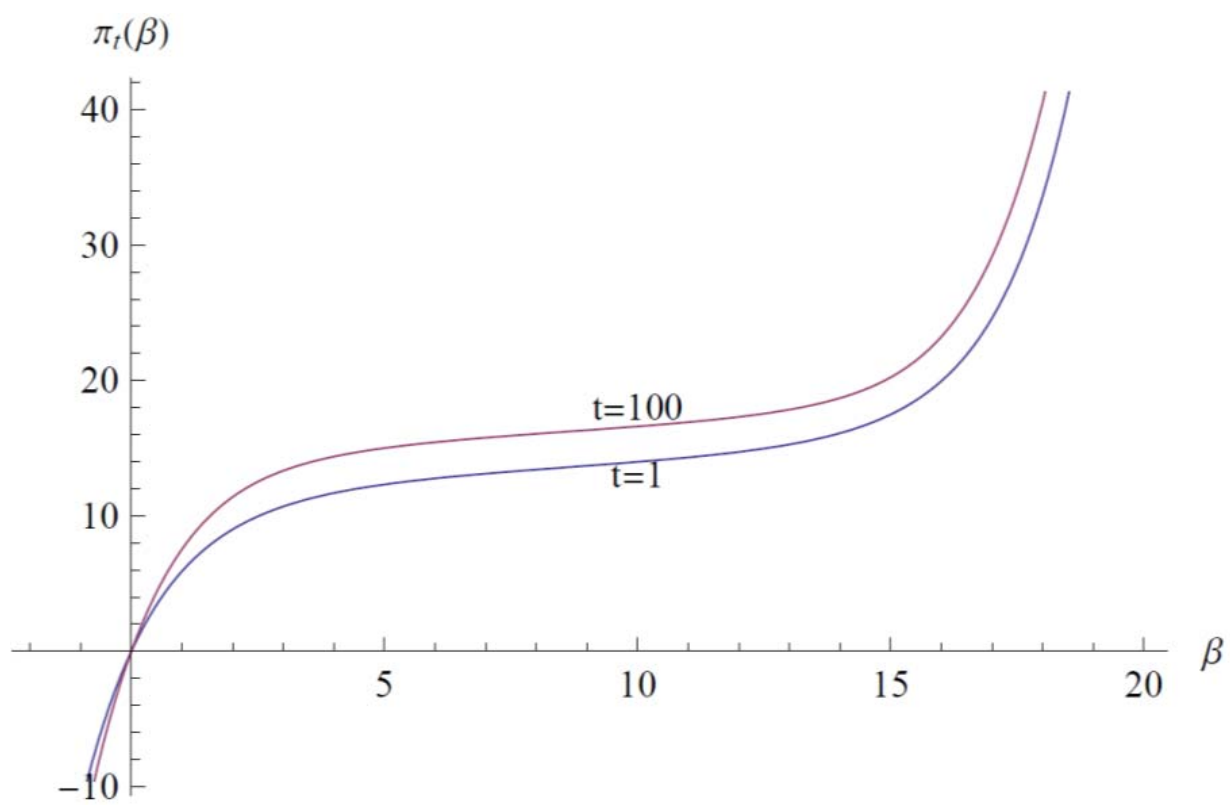

Figure 5: The risk premium $\pi_{t}(\beta)$ (in \%) as a function of $\beta$, for $t=1$ and $t=100$. The calibration is as in Figure 3.

It is interesting to observe that, as in the previous section, the risk premium $\pi_{t}(\beta)$ is usually not proportional to $\beta$. There are two reasons that explain this feature of asset prices in this context. Both are linked to the non-Gaussian nature of $G_{t}$. The first one comes from the fat tail induced 
by rare events. The second one comes from the uncertainty affecting the probability of rare events. Because the distribution of $G_{t}$ cannot be approximated by a Gaussian even for small maturities, the nonlinearity of the risk premium with respect to the beta of the asset is quite impressive, as can be seen in Figure 5. This nonlinearity appears to be a crucial element of asset prices in the context of rare catastrophic events at all maturities. Notice in particular that the risk premium is concave in beta in a wide range of this parameter. In Table 1, we provide some values for the discount rate of risky assets for different betas and maturities. We obtain that the term structure of the discount rate $\rho_{t}(\beta)$ is decreasing for all $\beta<2.92$, and is increasing for larger betas.

\begin{tabular}{l|cccc}
\hline \multicolumn{2}{c}{$t=1$} & $t=10$ & $t=100$ & $t=1000$ \\
\hline$\beta=-1$ & -11.0 & -12.7 & -16.5 & -16.9 \\
$\beta=0.0$ & 0.5 & -0.1 & -2.2 & -2.8 \\
$\beta=0.5$ & 3.9 & 3.6 & 2.2 & 1.6 \\
$\beta=1.0$ & 6.4 & 6.2 & 5.4 & 4.9 \\
$\beta=2.0$ & 9.5 & 9.4 & 9.2 & 8.9 \\
$\beta=5.0$ & 12.8 & 12.8 & 12.9 & 13.0 \\
\hline
\end{tabular}

Table 1: The discount rate $\rho_{t}(\beta)=r_{f t}+\pi_{t}(\beta)$ (in \%) calibrated with $\delta=3 \%, \gamma=4, n=2$, $\mu_{1}=2.5 \%, \sigma_{1}=2 \%, \mu_{2}=-39 \%$ and $\sigma_{2}=25 \%$. The probability of the catastrophic scenario $i=2$ is $1.2 \%$ or $2.2 \%$ with equal probabilities.

It may be tempting to approximate all terms of the form $\ln \left(x_{1}+p\left(x_{2}-x_{1}\right)\right)$ for $x=a(\beta), x=b$ or $x=d(\beta)$ that appears everywhere in this section either by its log-linearized version $(1-p) \ln \left(x_{1}\right)+p \ln \left(x_{2}\right)$, or by its first-order Taylor approximation $\ln \left(x_{1}\right)+p\left(x_{2}-x_{1}\right) / x_{1}$. However, because the distance between $x_{1}$ and $x_{2}$ is large in this model with catastrophes, these approximations are very poor. For example, the first-order Taylor approximation approach leads to the approximation $r_{f 0} \simeq-0.31 \%$, to be compared to the true value $r_{f 0}=0.52 \%$.

\section{Pricing projects with a non-constant beta}


Specification (7) is critical for our results, because it allows us to use the properties of CGF functions that appears in all pricing formulas used in this paper. Although specification (7) is restrictive, examining the pricing of a project satisfying it opens the path to examining the pricing of a much larger class of projects of the form

$$
F_{t}=\sum_{i=1}^{n} \alpha_{i t} F_{i t} \text {, with } F_{i t}=\xi_{i t} c_{t}^{\beta_{i}}
$$

where random variables $\xi_{i t}$ are independent of $c_{t}$. This project can be interpreted as a portfolio of $n$ different projects, project $i$ having a constant $\beta=\beta_{i}, i=1, \ldots, n$. Of course, the resulting “portfolio project” has a non-constant beta. By a standard arbitrage argument, the value of this portfolio is the sum of the values of each of its beta-specific components. We can thus rely on our results in this paper for the evaluation of projects with a non-constant beta. If $\rho_{t}\left(\beta_{i}\right)$ is the efficient discount rate to evaluate the $\beta_{i}$-component of the project, the global value of the project will be equal to

$$
\sum_{i=1}^{n} e^{-\rho_{t}\left(\beta_{i}\right) t} \alpha_{i t} E F_{i t}
$$

where we assumed without loss of generality that $E \xi_{i t}=1$. In other words, the discount factors rather than the discount rates - must be averaged to determine the discount factor to be used to evaluate the cash flows of the global portfolio. In the absence of parametric uncertainty, Weitzman (2012) examines the case in which the project under scrutiny is a portfolio of a risk free asset $\left(\beta_{1}=0\right)$ and of a risky project with a unit beta $\left(\beta_{2}=1\right)$.

\section{The beta of $\mathrm{CO}_{2}$ projects}

The purpose of this section is to heuristically derive a crude numerical estimate for term structure of discount rates to be used for the evaluation of climatic policies. To do this, we need to answer the following often overlooked question: What is the beta of investments whose main objective is to abate emissions of greenhouse gases? Let us consider a simple two-date version of the DICE model of Nordhaus (2008) and Nordhaus and Boyer (2000): 


$$
\begin{gathered}
T=\omega_{1} E \\
E=\omega_{2} Y-I_{0} \\
D=\theta_{1} T^{\theta_{2}} \\
Q=e^{-D} Y \\
C=\alpha Q
\end{gathered}
$$

All parameters of the model are assumed to be nonnegative. $T$ is the increase in temperature and $E$ is the emission of greenhouse gases from date 0 to date 1 . It is assumed in equation (57) that the increase in temperature is proportional to the emission of these gases. By equation (58), emissions are proportional to the pre-damage production level $Y$, but they can be reduced by investing $I_{0}$ in a green technology at date 0 . In equation (59), we assume that the damage $D$ is an increasing power function of the increase in temperature. Equation (60) defines damage $D$ as the logarithm of the ratio $Y / Q$ of pre-damage and post-damage production levels. ${ }^{10} \mathrm{We}$ hereafter refer to $D$ as the relative damage. Finally, consumption $C$ is proportional to the postdamage production $Q$. This model yields the following reduced form:

$$
C=\alpha Y \exp \left[-\theta_{1}\left(\omega_{1}\left(\omega_{2} Y-I_{0}\right)\right)^{\theta_{2}}\right]
$$

We consider the beta of a green investment $I_{0}$. Such an investment has the benefit to raise consumption in the future by

$$
\frac{\partial C}{\partial I_{0}}=\alpha Y \theta_{1} \theta_{2} \xi^{\theta_{2}}\left(\omega_{2} Y-I_{0}\right)^{\theta_{2}-1} \exp \left[-\theta_{1}\left(\omega_{1}\left(\omega_{2} Y-I_{0}\right)\right)^{\theta_{2}}\right]
$$

This is the future cash flow $F$ of the investment. We assume that this investment is marginal, so that our model can be rewritten as:

$$
\left\{\begin{array}{l}
C=\alpha Y \exp \left[-\theta_{1}^{*} Y^{\theta_{2}}\right] \\
F=\frac{\theta_{1}^{*} \theta_{2} \alpha}{\omega_{2}} Y^{\theta_{2}} \exp \left[-\theta_{1}^{*} Y^{\theta_{2}}\right],
\end{array}\right.
$$

\footnotetext{
${ }^{10}$ Equation (60) is traditionally expressed as $Q_{1}=\left(1-D_{1}\right) Y_{1}$. However, for high temperatures, this specification could lead to a negative after-damage production.
} 
where $\theta_{1}^{*}=\theta_{1} \omega_{1}^{\theta_{2}} \omega_{2}^{\theta_{2}}$ can be interpreted as a synthetic climate-sensitivity parameter.

A critical parameter for this model is $\theta_{2}$. When $\theta_{2}$ is equal to unity, the relative damage is just proportional to the change in temperature and in concentration of greenhouse gases. The absolute damage $Y-Q$ is thus convex in $Y$ in that case. When $\theta_{2}$ is larger than unity, the relative damage is itself convex, thereby bringing even more convexity to the absolute damage as a function of $Y$. Let us first assume that $\theta_{2}=1$. In that case, we can derive from system (64) that

$$
F=\theta_{1} \omega_{1} C
$$

Let us further assume that the only source of uncertainty is about the growth of pre-damage production. This implies that $\theta_{1} \omega_{1}$ is a constant. We can thus conclude in this case that the green investment project under scrutiny in this section satisfies condition (7) with $\beta=1$. This proves the following proposition.

Proposition 6: Consider the simplified integrated assessment model (57)-(61) with $\theta_{2}=1$ and without uncertainty about the climate parameter $v=\theta_{1} \omega_{1}$.Under this specification, any project whose benefits are to reduce emissions of greenhouse gases has a constant beta equaling unity.

The intuition of a positive beta in this model is as follows. When economic growth is high, more greenhouse gases are emitted in the atmosphere and the benefits of mitigation are large. Consumption and benefits covary positively in this model. However, this simple result raises two difficulties. First, although all experts in the field recognize the scarcity of evidence to infer $\theta_{2}$, most of them agree that the relation $D=f(T)$ should be convex, yielding $\theta_{2}>1$. Nordhaus and Boyer (2000) used $\theta_{2}=2,{ }^{11}$ whereas Cline (1992) used $\theta_{2}=1.3$. The Monte-Carlo simulations of the PAGE model used in the Stern (2007) Review draw $\theta_{2}$ from an asymmetric triangular probability density function with support in [1,3], giving a mean of about 1.8 (See Dietz, Hope and Patmore, 2007). Although there is no consensus on the value of this parameter, this suggests a more consensual beta somewhere between 1 and 2. Compared to the result in Proposition 6, a

\footnotetext{
${ }^{11}$ Nordhaus $(2007,2011)$ used a quadratic function, yielding a similar degree of convexity of the damage function in the relevant domain of increases in concentration.
} 
larger $\theta_{2}$ tends to increase the benefits of reducing emissions in good states (large $Y$ ), and to reduce them in bad states (low $Y$ ). Intuitively, this should raise the beta of green projects above unity. To see this observe that a local estimation of the beta from (7) can be obtained by fully differentiating system (64) with respect to $Y$. We obtained

$$
\beta \approx \frac{d \ln F / d Y}{d \ln C / d Y}=\theta_{2} \frac{1-\theta_{1}^{*} Y^{\theta_{2}}}{1-\theta_{1}^{*} Y}
$$

which is close to $\theta_{2}$ when $\theta_{2}$ is close to unity. Observe that the beta of the project is not constant when $\theta_{2}$ is not equal to unity. In other words, the expected benefit function conditional to $C$ is not a power function of $C$.

Second, when $v=\theta_{1} \omega_{1}$ is random, it will in general be correlated to $C$, as shown by the first equation in system (64). In that case, equation (65) cannot anymore be interpreted as describing a project with a unit beta. To illustrate this point, consider the extreme case where economic growth $Y$ is certain, together with $\omega_{1}, \omega_{2}$ and $\theta_{2}$, but the climate sensitivity parameter $\theta_{1}$ is uncertain. In that case, eliminating $\theta_{1}$ from system (64) yields

$$
F=\frac{\theta_{2}}{\omega_{2}} \frac{C}{Y} \ln \frac{\alpha Y}{C}
$$

In this case, cash flow $F$ is a deterministic function of $C$. Because it is not a power function, the beta of green projects is not constant in this specification. We can approximate it through the following formula:

$$
\beta \approx \frac{d \ln F / d \theta_{1}}{d \ln C / d \theta_{1}}=1-\frac{1}{D}
$$

with $D=\theta_{1}^{*} Y^{\theta_{2}}$. This typically yields a negative beta, which is large in absolute value. Indeed, if we assume a range of damages between $5 \%$ and $20 \%$ of the aggregate production, we obtain a beta in the range between -4 and -19 . In this story based on the uncertain climate sensitivity, a large sensitivity yields at the same time large damages, low consumption, and large benefits of mitigation. This explains the negative beta obtained under this specification. This story is similar to the one proposed by Sandsmark and Vennemo (2007) who claim that the beta of mitigation 
investments should be negative. ${ }^{12}$ Their argument is based on the climate variability as being the only source of fluctuation in the economy.

To sum up, the result presented in Proposition 6 suffers from two major deficiencies: The selected $\theta_{2}$ is too small, and it does not recognize that there is still much uncertainty about the sensitivity of the climate to an increase in concentration of greenhouse gases in the atmosphere. The difficulty is that improving the model to allow for $\theta_{2} \neq 1$ or for an uncertain climate sensitivity implies that the benefit $F$ cannot be written anymore as a power function of $C$ as in equation (7), or as a sum of power functions of $C$ as in equation (55).

We can conclude from this discussion that the beta of investments whose main benefits are a reduction of emissions of greenhouse gases is non-constant. Its average level is determined by the relative intensity of two sources of uncertainty, the one coming from the future economic prosperity, and the one due to the unknown intensity of the climatic problem. We believe that the economic source of variability has an order of magnitude larger than the climatic source of variability. When the annual growth rate of the economy varies between $0 \%$ and $3 \%$, aggregate consumption in 100 years is between $0 \%$ and $1800 \%$ larger than today. This should be compared to climate damages for this time horizon which are usually estimated between $0 \%$ and $5 \%$ (see for example the Stern Review, 2007). To make this argument more concrete, let us consider the calibration of the simple above model as described in Table 2.

\begin{tabular}{|c|c|c|}
\hline Variable & Value & Remark \\
\hline$t$ & 50 years & Time horizon between dates 0 and 1 . \\
\hline$Y=\mathrm{e}^{\sum_{i=1}^{t} x_{i}}$ & $\begin{array}{l}x_{i} i i d \sim N\left(\mu, \sigma^{2}\right) \\
\mu=1.5 \%, \sigma=4 \%\end{array}$ & $\begin{array}{l}Y_{0} \text { is normalized to unity. The growth rate of production } \\
\text { follows a normal random walk. }\end{array}$ \\
\hline$\omega_{2}$ & 1 & Normalization \\
\hline$\omega_{1}$ & 0.45 & $\begin{array}{l}\text { This implies that the expected increase in temperature in } \\
\text { the next } 50 \text { years equals } \xi E Y=1^{\circ} \mathrm{C} \text {. }\end{array}$ \\
\hline$\theta_{2}$ & 1.5 & Center of the "consensus interval” [1,2]. \\
\hline
\end{tabular}

\footnotetext{
${ }^{12}$ They obtain $\beta=-0.004$, which is much closer to zero that what we obtain here. However, notice that these authors consider another definition of the beta, which is equal to the ratio of the covariance of $\left(C_{1}, F_{1}\right)$ to the variance of $C_{1}$. In our model, the beta is equal to the ratio of the covariance of $\left(\ln C_{1}, \ln F_{1}\right)$ to the variance of $\ln C_{1}$.
} 


$\begin{array}{lll}\theta_{1} & \sim U[0 \%, 5 \%] & \begin{array}{l}\text { This means that the damage at the average temperature } \\ \text { increase of } 1^{\circ} \mathrm{C} \text { is uniformly distributed on }[0 \%, 5 \%] \text { of } \\ \text { pre-damage production. }\end{array} \\ \alpha & 0.75 & \text { Consumption equals } 75 \% \text { of post-damage production. }\end{array}$

Table 2: Calibration of the two-date IAM model

Using the Monte-Carlo method, we generated 50000 independent random selections of the pair $\left(Y, \theta_{1}\right)$ and the corresponding outcomes in terms of aggregate consumption $C$ and benefits of mitigation $F$, as defined by system (64). Using these data, we regressed $\ln F$ on $\ln C$. The OLS estimation of the beta equals $\hat{\beta}=1.32$ with a standard deviation of $0.016{ }^{13}$ Because of the predominance of the uncertainty about economic growth in the long run, and because of the convexity of the cost function, there is a positive correlation between economic growth and the benefits of mitigation, yielding a large positive beta. This is in line with the recent results by Nordhaus (2011), in which the author summarizes the outcome of Monte-Carlo simulations of the much more sophisticated RICE-2011 model with 16 sources of uncertainty: “ Those states in which the global temperature increase is particularly high are also ones in which we are on average richer in the future."

In Figure 6, we draw the term structures of discount rates prevailing for $\beta=1.32$ in three different phases of the macroeconomic cycle under the calibration used in Section 4. The discount rate to be used for super-long maturities is around $4.6 \%$, whereas the short-term discount rate fluctuates along the business cycle from around $1.3 \%$ when the expected instantaneous trend is $1.2 \%$ per annum below its historical mean, to around $6 \%$ when the expected instantaneous trend is $1.2 \%$ above its historical mean.

\footnotetext{
${ }^{13}$ Increasing the degree of uncertainty affecting $\theta_{l}$ has a sizeable impact on this estimation. For example, if we replace the assumption that the interval $[0 \%, 5 \%]$ on which it is uniformly distributed by $[0 \%, 10 \%]$, the OLS estimation of the beta goes down to $1.14 \%$. However, it is hard to imagine damages amounting to $10 \%$ of the world production due to a $1^{\circ} \mathrm{C}$ increase in temperature.
} 


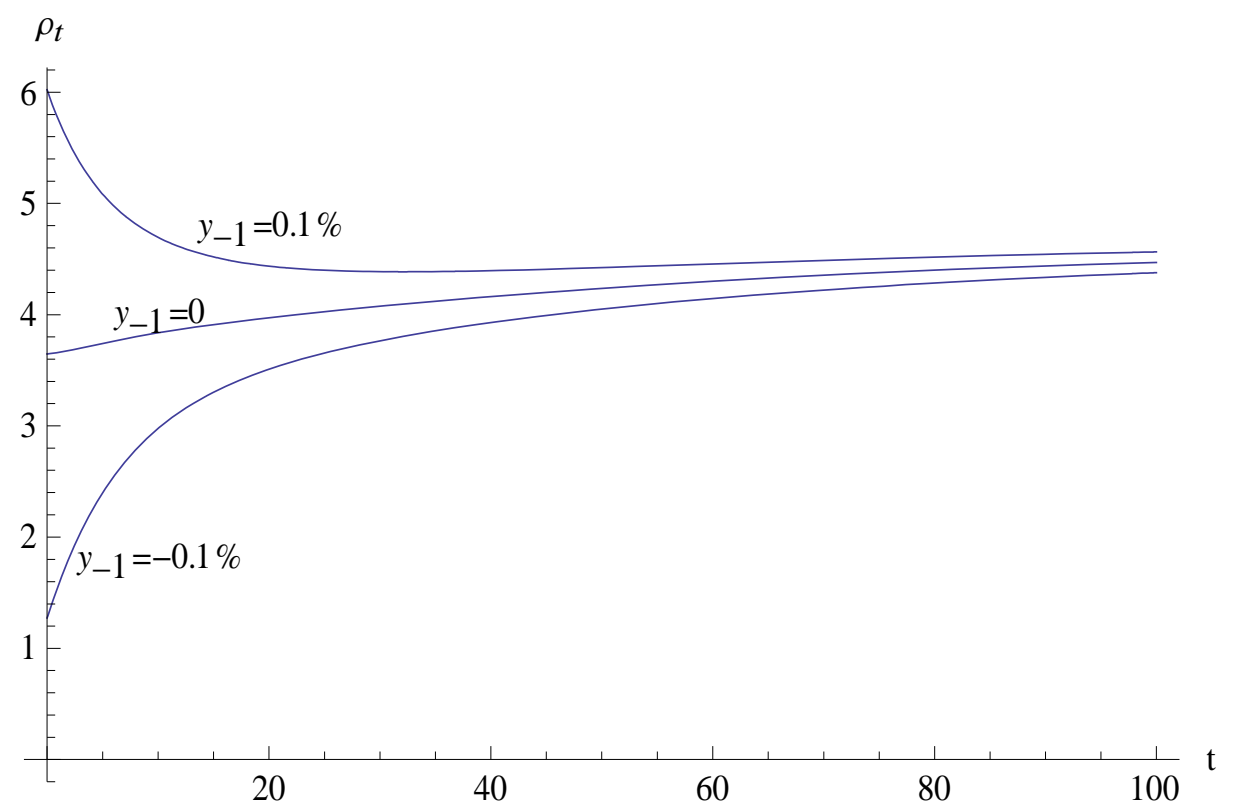

Figure 6: The discount rate (in \% per year) as a function of the maturity (in years) for $\beta=1.32$ in different phases of the cycle. Equation (40) is calibrated with $\delta=0, \gamma=2, \sigma_{x}=0.0078$, $\sigma_{y}=0.00034, \phi=0.979$, and two equally likely trends $\mu_{1}=0.0005$ and $\mu_{2}=0.0025$.

\section{Concluding remarks}

By focusing on the riskiness of future benefits and costs, this paper contributes to the debate on the discount rate for climate change in several directions. Following Weitzman (2007) and Gollier (2008), we explicitly take into account of the deep parametric uncertainties that affect our economic growth in the distant future. Our benchmark model is such that the underlying growth rate of consumption is Gaussian, but with unknown drift and volatility. Our main messages in this framework are as follows. First, we showed that the shape of the term structure of discount rates for risky projects is determined by the relative intensity of a precautionary effect that pushes towards a decreasing term structure, and of a risk aversion effect that pushes towards an increasing term structure. Under some weak restrictions on the distribution of the uncertain parameters, the term structure is decreasing or increasing depending upon whether the beta of the project is respectively smaller or larger than half the relative risk aversion of the representative agent. Second, we showed that the risk premium associated to a project is generally not proportional to its beta, which implies that knowing the aggregate risk premium and the project's 
beta is not enough to compute the project-specific risk premium. We derived simple formulas to compute the project-specific risk premium as a function of the project's beta.

We have also examined a model à la Barro (2006) in which the growth rate of consumption has fat tails, because of a small probability of a macroeconomic catastrophe that is added to the otherwise Gaussian business-as-usual growth process. The recognition of the intrinsic ambiguity that affects the frequency of catastrophes implies that the risk free discount rate and the aggregate risk premium should be respectively decreasing and increasing.

Finally, we have shown that there are reasons to believe that the beta of projects whose main benefits are to reduce emissions of greenhouse gases is relatively large, around 1.3. This allows us to conclude that the discount rates to be used to evaluate public policies to fight climate change should be increasing with respect to maturities. Given the current global economic crisis in the western world, following Figure 5, we are in favor of using a real discount rate for climate change around $1.3 \%$ for short horizons, up to $4.6 \%$ for maturities exceeding 100 years.

A word of caution should be added to this conclusion. The use of price signals like discount rates and risk premia is possible only for investment projects that are marginal, i.e., for actions that do not affect expectations about the growth of the economy. The reader should be aware that this assumption does not hold when considering the global strategy to fight climate change. When thinking globally, one needs to take into account the general equilibrium effects that the chosen strategy will have on the stochastic growth process, hence on the discount rates that are used to evaluate this strategy. The right evaluation approach for global projects relies on the direct measure of the impact of the global action on the intergenerational social welfare function, as done for example in Stern (2007) and Nordhaus (2008). 


\section{Appendix: Proof of Proposition 3}

If we define $y=\mu-m_{1}^{\mu}$, we can rewrite equation (26) as follows:

$$
\rho_{t}(\beta)=\rho_{0}(\beta)-\frac{1}{t} \ln \frac{E e^{(\beta-\gamma) y}}{E e^{\beta y}},
$$

Fully differentiating this equation twice with respect to $t$ and $\gamma$ yields

$$
\begin{aligned}
\frac{\partial^{2} \rho_{t}(\beta)}{\partial \gamma \partial t} & =(\beta-\gamma)\left(\frac{E y^{2} e^{t(\beta-\gamma) y}}{E e^{t(\beta-\gamma) y}}-\left(\frac{E y e^{t(\beta-\gamma) y}}{E e^{t(\beta-\gamma) y}}\right)^{2}\right) \\
& =(\beta-\gamma)\left[E z^{2}-(E z)^{2}\right],
\end{aligned}
$$

where $z$ is the random variable which takes value $z_{\theta}=\mu_{\theta}-m_{1}^{\mu}$ under the distorted probability distribution $P_{t}(\theta)$ such that

$$
d P_{t}(\theta)=\frac{e^{t(\beta-\gamma)\left(\mu_{\theta}-m_{1}^{\mu}\right)}}{E e^{t(\beta-\gamma)\left(\mu-m_{1}^{\mu}\right)}} d P(\theta)
$$

Because the bracketed term in the RHS of (70) is always positive, we have that $\partial \rho_{t}(\beta) / \partial t$ is decreasing in $\gamma$ for all $\beta \in]-\infty, \gamma]$. Because it vanishes at $\beta=\gamma / 2$, we obtain that $\partial \rho_{t}(\beta) / \partial t$ is negative for all $\beta<\gamma / 2$, and it is positive for all $\beta \in[\gamma / 2, \gamma]$. 


\section{References}

Arrow, K.J., and R.C. Lind, (1970), Uncertainty and the evaluation of public investment decision, American Economic Review, 60, 364-378.

Backus, D., K. Chernov, and I. Martin, (2011), Disasters implied by equity index options, Journal of Finance (66), 1969-2012.

Bansal, R., and A. Yaron, (2004), Risks For the Long Run: A Potential Resolution of Asset Pricing Puzzles, Journal of Finance, 59, 1481-1509.

Barro, R.J., (2006). Rare Disasters and Asset Markets in the Twentieth Century, Quarterly Journal of Economics, 121, 823-866.

Barro, R.J., (2009), Rare disasters, asset prices, and welfare costs, American Economic Review, 99, 243-264.

Billingsley, P., (1995), Probability and Measure, Wiley, New York.

Cline, W.R., (1992), The Economics of Global Warming, Institute for International Economics, Washington.

Dietz, S., C. Hope and N. Patmore, (2007), Some economics of 'dangerous' climate change: Reflections on the Stern Review, Global Environmental Change, 17, 311-325.

Drèze, J.H. and F. Modigliani, (1972), Consumption decisions under uncertainty, Journal of Economic Theory, 5, 308-335.

European Insurance and Occupational Pensions Authority, (2012), Draft technical specifications QIS of EIOPA's advice on the review of the IORP directive: Consultation paper, EIOPA-CP-12003, 15 June 2012.

Gollier, C., (2002), Time horizon and the discount rate, Journal of Economic Theory, 107, 463473.

Gollier, C., (2008), Discounting with fat-tailed economic growth, Journal of Risk and Uncertainty, 37, 171-186. 
Gollier, C., (2011), Le calcul du risque dans les investissements publics, Centre d'Analyse Stratégique, Rapports \& Documents n³6, La Documentation Française.

Gollier, C., (2012), Pricing the planet's future: The economics of discounting in an uncertain world, Princeton University Press, Princeton.

Kimball, M.S., (1990), Precautionary savings in the small and in the large, Econometrica, 58, 53-73.

Lucas, R., (1978), Asset prices in an exchange economy, Econometrica, 46, 1429-46.

Martin, I., (2012), Consumption-based asset pricing with higher cumulants, Review of Economic Studies, forthcoming.

Mehra, R. and E. Prescott, (1985), The Equity Premium: A Puzzle, Journal of Monetary Economics, 10, 335-339.

Newell, R. and W. Pizer, (2003), Discounting the Benefits of Climate Change Mitigation: How Much Do Uncertain Rates Increase Valuations? Journal of Environmental Economics and Management, 46 (1), 52-71.

Nordhaus, W.D., (2008), A Question of Balance: Weighing the Options on Global Warming Policies, New Haven: Yale University Press.

Nordhaus, W.D., (2011), Estimates of the social cost of carbon: Background and results from RICE2011 model, NBER WP17540, http://www.nber.org/papers/w17540.

Nordhaus, W.D., and J. Boyer, (2000), Warming the World: Economic Models of Global Warming, Cambridge, MA: MIT Press.

Office of Management and Budget, (2003), Circular N. A-4 To the Heads of Executive Department Establishments, Subject: Regulatory Analysis. Washington: Executive Office of the President.

Sandsmark, M., and H. Vennemo, (2007), A portfolio approach to climate investments: CAPM and endogeneous risk, Environmental and Resource Economics, 37, 681-695.

Stern, N., (2007), The Economics of Climate Change: The Stern Review, Cambridge University Press, Cambridge. 
Weil, P., (1989), The equity premium puzzle and the risk free rate puzzle, Journal of Monetary Economics, 24, 401-21.

Weitzman, M.L., (1998), Why the far-distant future should be discounted at its lowest possible rate?, Journal of Environmental Economics and Management, 36, 201-208.

Weitzman, M.L., (2001), Gamma discounting, American Economic Review, 91, 260-271.

Weitzman, M. L., (2007), Subjective expectations and asset-return puzzle, American Economic Review, 97, 1102-1130.

Weitzman, M. L., (2009), On Modeling and Interpreting the Economics of Catastrophic Climate Change, Review of Economics and Statistics, 91 (1), 1-19.

Weitzman, M.L., (2010), Risk-adjusted gamma discounting, Journal of Environmental Economics and Management, 60, 1-13.

Weitzman, M.L., (2012), Rare disasters, tail-hedged investments and risk-adjusted discount rates, NBER WP18496. 